\title{
GLOBAL SMOOTH ION DYNAMICS IN THE EULER-POISSON SYSTEM
}

\author{
YAN GUO AND BENOIT PAUSADER
}

\begin{abstract}
A fundamental two-fluid model for describing dynamics of a plasma is the Euler-Poisson system, in which compressible ion and electron fluids interact with their self-consistent electrostatic force. Global smooth electron dynamics were constructed in Guo [9] due to dispersive effect of the electric field. In this paper, we construct global smooth irrotational solutions with small amplitude for ion dynamics in the Euler-Poisson system.
\end{abstract}

\section{Contents}

1. Introduction and Formulation

1.1. The ionic Euler-Poisson system

1.2. Presentation of the paper

1.3. Notations and preliminary results

2. Linear Decay

3. Normal form transformation

4. The $L^{2}$-type norm

4.1. The Energy estimate

4.2. The $H^{-1}$-norm

5. Bilinear Multiplier Theorem

5.1. A general multiplier theorem

5.2. Multiplier Analysis

6. The $L^{10}$ Bound and end of the proof 29

6.1. Estimating the $L^{10}$ bound 29

6.2. End of the proof 32

References 32

\section{Introduction AND Formulation}

1.1. The ionic Euler-Poisson system. The "two-fluid" models in plasma physics describe dynamics of two separate compressible fluids of ions and electrons interacting with their self-consistent electromagnetic field. Many famous nonlinear dispersive PDE, such as Zakharov's equation, nonlinear Schrödinger equations, as well as KdV equations, can be formally derived from "two-fluid" models under various asymptotic limits. In the absence of the magnetic effects, the fundamental two-fluid model for describing the dynamics of a plasma is given by the following 
Euler-Poisson system

$$
\begin{aligned}
\partial_{t} n_{ \pm}+\nabla \cdot\left(n_{ \pm} v_{ \pm}\right) & =0 \\
n_{ \pm} m_{ \pm}\left(\partial_{t} v_{ \pm}+v_{ \pm} \cdot \nabla v_{ \pm}\right)+T_{ \pm} \nabla n_{ \pm} & =e n_{ \pm} \nabla \phi \\
\Delta \phi & =4 \pi e\left(n_{+}-n_{-}\right) .
\end{aligned}
$$

Here $n_{ \pm}$are the ion $(+)$and electron density $(-), v_{ \pm}$are the ion $(+)$and electron $(-)$ velocity, $m_{ \pm}$are the masses of the ions $(+)$and electrons $(-), T_{ \pm}$are their effective temperatures, and $e$ is the charge of an electron. The self-consistent electric field $\nabla \phi$ satisfies the Poisson equation. The Euler-Poisson system describes rich dynamics of a plasma. Indeed, even at the linearized level, there are electron waves, ion acoustic waves in the Euler-Poisson system. Despite its importance, there has been few mathematical study of its global solutions in $3 \mathrm{D}$. This stems from the fact that the Euler-Poisson system belongs to the general class of hyperbolic conservation laws with zero dissipation, for which no general mathematical framework for construction of global in-time solutions exists in 3D. In fact, as expected [10, solutions of the Euler-Poisson system with large amplitude in general will develop shocks.

However, unlike the pure Euler equations, shock formation for solutions of the Euler-Poisson system with small amplitude has remained open. In Guo 9], the first author studied a simplified model of the Euler-Poisson system for an electron fluid:

$$
\begin{aligned}
\partial_{t} n_{-}+\nabla \cdot\left(n_{-} v_{-}\right) & =0 \\
n_{-} m_{-}\left(\partial_{t} v_{-}+v_{-} \cdot \nabla v_{-}\right)+T_{-} \nabla n_{-} & =e n_{-} \nabla \phi \\
\Delta \phi & =4 \pi e\left(n_{-}-n_{0}\right) .
\end{aligned}
$$

In this model, the ions are treated as immobile and only form a constant charged background $n_{0}$. Surprisingly, it was observed [9] that the linearized Euler-Poisson system for the electron fluid is the Klein-Gordon equation, due to plasma oscillations created by the electric field $\phi$. In this case, the dispersion relation reads

$$
\omega(\xi) \backsim \sqrt{1+|\xi|^{2}}
$$

Such a "Klein-Gordon" effect led to construction of smooth irrotational electron dynamics with small amplitude for all time. This is in stark contrast to the pure Euler equations for neutral fluids where the dispersion relation reads

$$
\omega(\xi) \backsim|\xi|,
$$

in which shock waves can develop even for small smooth initial data (see Sideris 23]). It is the dispersive effect of the electric field that enhances the linear decay rate and prevents shock formation. The natural open question remains: does such a dispersive effect exist generally ? If so, can it prevent shock formation for the general Euler-Poisson system (1.1) ?

In the current paper, we make another contribution towards answering this question. We consider another (opposite) asymptotic limit of the original Euler-Poisson system (1.1) for the ion dynamics. It is well-known that $\frac{m_{-}}{m_{+}}<<1$ in all physical situations. By letting the electron mass $m_{-}$go to zero, we formally obtain $T_{-} \nabla n_{-}=e n_{-} \nabla \phi$ and the famous Boltzmann relation

$$
n_{-}=n_{0} \exp \left(\frac{e \phi}{T_{-}}\right)
$$

for the electron density ( $n_{0}$ is a constant). Such an important relation (1.3) can also be verified through arguments from kinetic theory, see Cordier and Grenier $[3]$. 
We then obtain the well-known ion dynamic equations as

$$
\begin{aligned}
\partial_{t} n_{+}+\nabla \cdot\left(n_{+} v_{+}\right) & =0 \\
n_{+} m_{+}\left(\partial_{t} v_{+}+v_{+} \cdot \nabla v_{+}\right) & =-T_{+} \nabla n_{+}-n_{+} e \nabla \phi \\
\Delta \phi & =4 \pi e\left(n_{0} \exp \left(\frac{e \phi}{T_{-}}\right)-n_{+}\right) .
\end{aligned}
$$

We also assume that

$$
\operatorname{curl}(v(0))=0 .
$$

It is standard that the condition (1.5) is preserved by the flow. As a matter of fact, non irrotational flow leads to creation of a non-vanishing magnetic field, which is omitted in the Euler-Poisson system but retained in a more general Euler-Maxwell system [1. The linear dispersion relation for (1.4) behaves like

$$
p(\xi) \equiv|\xi| \sqrt{\frac{2+|\xi|^{2}}{1+|\xi|^{2}}} \equiv|\xi| q(|\xi|)
$$

which is much closer to the wave dispersion $\omega(\xi)=|\xi|$ than to the Klein-Gordon one, $\omega(\xi)=\sqrt{1+|\xi|^{2}}$ (in particular note this dispersion relation behaves near 0 as in the Schrödinger case, whereas in our dispersion relation $p$ remains very similar to that of the wave's). Intuitively, one might expect formation of singularity for (1.4) as in the pure Euler equations. Nevertheless, we demonstrate that small smooth irrotational flows exist globally in time, and there is no shock formation. Without loss of generality, we study the global behavior of irrotational perturbations of the uniform state

$$
\left[n_{+}, v_{+}\right]=\left[n_{0}+\rho, v\right] .
$$

We use two important norms defined as follows:

$$
\begin{aligned}
\|u(x)\|_{Y} & =\left\||\nabla|^{-1} u\right\|_{H^{2 k+1}}+\|u\|_{W^{k+\frac{12}{5}, \frac{10}{9}}} \\
\|u(t, x)\|_{X} & =\sup _{t}\left(\left\||\nabla|^{-1}(1-\Delta)^{k+\frac{1}{2}} u(t)\right\|_{L^{2}}+(1+t)^{\frac{16}{15}}\left\|(1-\Delta)^{\frac{k}{2}} u(t)\right\|_{L^{10}}\right)
\end{aligned}
$$

for $k \geq 5$.

Here, we keep $k$ as a parameter to emphasize the fact that smoother initial data lead to smoother solutions. Hidden in the $X$-norm is a statement about preservation of regularity of $(\rho, v)$. Our main result is the following

Theorem 1.1. There exists $\varepsilon>0$ such that any initial perturbation $\left(n_{0}+\rho_{0}, v_{0}\right)$ satisfying (1.5) $\nabla \times v_{0}=0$ and $\left\|\rho_{0}\right\|_{Y}+\left\|v_{0}\right\|_{Y} \leq \varepsilon$ leads to a global solution $\left(n_{0}+\rho, v\right)$ of (1.4) with

$$
\|\rho\|_{X}+\|v\|_{X} \leq 2 \varepsilon
$$

In particular, the perturbations $\rho$ and $v$ decay in $L^{\infty}$.

Together with earlier result in Guo 9, global smooth potential flows with small velocity exist for two opposite scaling limits of (1.1). This is a strong and exciting indication that shock waves of small amplitude should be absent for the full EulerPoisson system (1.1), at least in certain physical regimes. Our method developed in this paper should be useful in the future study of (1.1).

There have been a lot of mathematical studies of various aspects of the EulerPoisson system for a plasma. Texier [26, 27] studied the Euler-Maxwell system and its approximation by the Zakharov equations. Wang and Wang [29] constructed 
large BV radially symmetric solutions outside the origin. In Liu and Tadmor [16, 17, threshold for singularity formation has been studied for the Euler-Poisson system with $T_{ \pm}=0$ in one and two dimensions. In Feldman, Ha and Slemrod 44, 5, plasma sheath problem of the Euler-Poisson system was investigated. In Peng and Wang [20, Euler-Poisson system is derived from Euler-Maxwell system with a magnetic field. Quasi-neutral limit in the Euler-Poisson system was studied in Cordier and Grenier [3] and Peng and Wang [21. When $n_{+}$is replaced by a doping profile and a momentum relaxation is present, the Euler-Poisson system describes electron dynamics in a semiconductor device. There has been much more mathematical study of such a model, for which we only refer to Chen, Jerome and Wang 1] and the references therein.

1.2. Presentation of the paper. For notational simplicity, we let $n_{0}=e=T_{+}=$ $T_{-}=1$ in (1.4) throughout the paper. Even though the ion dynamics system (1.4) is the most natural system to further understand the dispersive effects in the full Euler-Poisson system (1.1), it has been remained an open problem to construct global smooth solutions until now ever since the work of [9], due to much more challenging mathematical difficulties than in the case of the electron Euler-Poisson equation (1.2) studied by Guo 9 .

The first difficulty is to understand the time decay rate of the linearized ion dynamics equation:

$$
\partial_{t t} \rho-\Delta \rho-\Delta(-\Delta+1)^{-1} \rho=0 .
$$

whose solutions are given by the operator $e^{ \pm p(|\nabla|) t}$ with p given by (1.6). Unlike the linearized electron equations studied in [9], there is no direct study of the linear decay of such a system. Only recently [11, time-decay rate for general dispersive equations has been carried out in detail with asymptotic conditions near low frequency $|\xi|=0$ and high frequency $|\xi|=\infty$. Interestingly, any phase $p(\xi)$ which is not exactly the phase function of the wave equation $p(\xi)=|\xi|$ commands a decay rate better than $\frac{1}{t}$. We are able to employ this result together with a stationary phase analysis near the inflection point of $p(\xi)$ to obtain a decay rate of $\frac{1}{t^{4 / 3}}$, which is between the wave and the Klein-Gordon equations. A consequence of the linear estimates of Section 2 is that

$$
\left\|e^{i t p(|\nabla|)} \alpha_{0}\right\|_{X} \lesssim\left\|\alpha_{0}\right\|_{Y}
$$

The main mathematical difficulty in this paper stems from bootstrapping the linear decay into a construction of global solutions to the nonlinear problem. Based on very recent new techniques of harmonic analysis in the study of dispersive PDE by Germain, Masmoudi and Shatah [6, 7, 8, Gustafson, Nakanishi and Tsai [12, 13, Shatah [22, we follow a new set-up for normal form transformation in 6, 13, 22, Using that $\nabla \times v \equiv 0$, we can introduce a pair of complex valued new unknowns:

$$
\alpha_{1}=\rho-\frac{i}{q(|\nabla|)} \mathcal{R}^{-1} v, \text { and } \alpha_{2}=\rho+\frac{i}{q(|\nabla|)} \mathcal{R}^{-1} v
$$

for $q$ defined in (1.6), where $\mathcal{R}=\nabla|\nabla|^{-1}$ stands for the Riesz transform, and $v=\nabla \psi, \mathcal{R}^{-1} v \equiv|\nabla| \psi$. After the normal form transformation (3.14), it suffices for 
us to control

$$
\begin{aligned}
& \hat{\alpha}(t) \backsim \int_{\mathbb{R}^{3}} \frac{m(\xi, \eta)}{\Phi_{1}(\xi, \eta)} \hat{\alpha}(\xi-\eta) \hat{\alpha}(\eta) d \eta \\
& +\int_{0}^{t} \int_{\mathbb{R}^{6}} e^{i(t-s) p(|\xi|)} \frac{m(\xi, \eta) m(\eta, \zeta)}{\Phi_{1}(\xi, \eta)} \hat{\alpha}(\xi-\eta) \hat{\alpha}(\eta-\zeta) \hat{\alpha}(\zeta) d \eta d \zeta d s .
\end{aligned}
$$

where $m$ denotes a generic multiplier given by (3.12). This is well defined in views of (1.5) and

$$
\Phi_{1}=p(|\xi|)-p(|\xi-\eta|)-p(|\eta|) .
$$

In the Klein-Gordon case, the phase is bounded away from zero so there is no singularity. However, for $\Phi_{1}$, there is a significant zero set when $|\xi-\eta||\eta|=0$, (see Lemma 5.3) and there is no "null form" structure to cancel with the multiplier $m$. We first observe that

$$
m(\xi, \eta) m(\eta, \zeta) \backsim|\xi||\eta|
$$

We then make use of such a structure to form a locally bounded multiplier

$$
\mathcal{M}_{1}=\frac{|\xi||\xi-\eta||\eta|}{\Phi_{1}(\xi, \eta)} \lesssim 1
$$

This process introduces a singular term $\frac{\hat{\alpha}(\xi-\eta)}{|\xi-\eta|}$, which will be controlled in a separate fashion by the $H^{-1}$ norm in our norm $\|\cdot\|_{X}$. We believe that including this $H^{-1}$ control in the norm should work equally well for equations with nonlinearity which has perfect spatial derivatives.

Even though $\mathcal{M}_{1}$ is locally bounded, it is very difficult to employ classical bilinear estimate such as Coifman-Meyer Theorem 2 to control (1.9). This is due to the anisotropic nature of $\mathcal{M}_{1}$ since $|\eta|$ can be very small with respect to $|\xi-\eta|$. Instead, we make use of a very recent multiplier estimate by Gustafson, Nakanishi and Tsai 13. It is important to use $L^{10}$-norms as a proxy for the $L^{\infty}$-norm for which our degenerate multipliers are not well-suited (we need an $L^{p}$-norm with $p<12$ ). The optimal Sobolev regularity for $\mathcal{M}_{1} \in L_{\xi}^{\infty}\left(\dot{H}_{\eta}^{5 / 4-\varepsilon}\right) \cap L_{\eta}^{\infty}\left(\dot{H}_{\xi}^{5 / 4-\varepsilon}\right)$ is crucial in applying such an estimate to obtain $L^{10}$ decay, and its proof is particularly delicate for small frequencies. We split the phase space and make a careful interplay between angles and the lengths of $\xi, \eta, \xi-\eta$. We also make use of Littlewood-Paley decomposition and interpolation to obtain a sharp Sobolev estimate for $\mathcal{M}_{1}$. On the other hand, to reduce the requirement of number of derivatives in our norm $X$, we also need to show a stronger estimate $\mathcal{M}_{1} \in L_{\xi}^{\infty}\left(\dot{H}_{\eta}^{3 / 2-\varepsilon}\right) \cap L_{\eta}^{\infty}\left(\dot{H}_{\xi}^{3 / 2-\varepsilon}\right)$ for large frequencies.

This paper is organized as follows: in Section 2 we study the relevant linear dispersive equation. In Section [3, we introduce our normal form transformation. In Section 4, we get an estimate on the $L^{2}$-part of the $X$ norm using the energy method. In Section 5 we state and prove the relevant multiplier estimate we need in order to control our bilinear terms. Finally, in Section 6, we control the high integrability part of the norm, and finish the analysis to obtain global solutions with small initial data in Theorem 1.1

1.3. Notations and preliminary results. We work in dimension $n=3$, although we state some results in arbitrary dimension $n$. We introduce

$$
\langle a\rangle=\sqrt{1+a^{2}} .
$$


We write $A \lesssim B$ to signify that there exists a constant $C$ such that $A \leq C$. We write $A \simeq B$ if $A \lesssim B \lesssim A$. Our phases and some multiplier are radial functions, and in some cases we might abuse notations and write, for a radial function $f$, $f(x)=f(|x|)$.

Our multipliers are estimated using the homogeneous Sobolev norm defined for $0 \leq s<n / 2$ by

$$
\|f\|_{\dot{H}^{s}}=\left\||\nabla|^{s} f\right\|_{L^{2}}
$$

where $|\nabla|$ is defined by $\mathcal{F}(|\nabla| f)(\xi)=|\xi| \hat{f}(\xi)$.

We will also use the Littlewood-Paley multipliers $P_{N}$ defined for dyadic numbers $N \in 2^{\mathbb{Z}}$ by

$$
P_{N} g=\mathcal{F}_{\xi}^{-1} \varphi\left(\frac{\xi}{N}\right) \mathcal{F}_{\xi} g
$$

where $\varphi \in C_{c}^{\infty}\left(\mathbb{R}^{n}\right)$ is such that

$$
\forall \xi \neq 0, \quad \sum_{N \in 2^{\mathbb{Z}}} \varphi\left(\frac{\xi}{N}\right)=1
$$

and for later use, we also introduce a function $\chi \in C_{c}^{\infty}\left(\mathbb{R}^{n}\right)$ such that $\chi \varphi=\varphi$. An important estimate on these Littlewood-Paley multipliers is the Bernstein inequality:

$$
\left\||\nabla|^{ \pm s} P_{N} f\right\|_{L^{p}} \lesssim s N^{ \pm s}\left\|P_{N} f\right\|_{L^{p}} \lesssim_{s} N^{ \pm s}\|f\|_{L^{p}}
$$

for all $s \geq 0$, and all $1 \leq p \leq \infty$, independently of $f, N$, and $p$, where $|\nabla|^{s}$ is the classical fractional differentiation operator.

We will also need the two following product estimates:

Lemma 1.1. Let $\tau$ be a multi-index of length $|\tau|$ and $\gamma<\tau$, then for all $u \in$ $C_{c}^{\infty}\left(\mathbb{R}^{n}\right)$ and all $\delta>0$, there holds that

$$
\left\|D^{\tau-\gamma} u D^{\gamma} \partial_{j} u\right\|_{L^{2}} \lesssim_{\delta}\|u\|_{W^{1+\delta, \infty}}\|u\|_{H^{|\tau|}} \lesssim\|u\|_{W^{2,10}}\|u\|_{H^{|\tau|}}
$$

Proof. We first note that without loss of generality, we may assume that $|\gamma|+$ $1,|\tau|-|\gamma| \geq 2$, otherwise Hölder's inequality gives the result. We use a simple paradifferential decomposition, in other words, we write

$$
\begin{aligned}
D^{\tau-\gamma} u D^{\gamma} \partial_{j} u & =\left(\sum_{M \sim N}+\sum_{M / N \leq 1 / 16}+\sum_{N / M \leq 1 / 16}\right)\left(P_{M} D^{\tau-\gamma} u\right)\left(P_{N} D^{\gamma} \partial_{j} u\right) \\
& =R+T_{1}+T_{2}
\end{aligned}
$$

where $M$ and $N$ are dyadic numbers. We first estimate $R$ as follows using Bernstein properties and in particular the fact that

$$
\left\|P_{N} u\right\|_{L^{\infty}} \lesssim \min \left(1, N^{-1-\delta}\right)\|u\|_{W^{1+\delta, \infty}}
$$


we get that with the Cauchy Schwartz inequality that

$$
\begin{aligned}
\|R\|_{L^{2}} & \lesssim \sum_{M \sim N}\left\|P_{M} D^{\tau-\gamma} u P_{N} D^{\gamma} \partial_{j} u\right\|_{L^{2}} \\
& \lesssim \sum_{M \sim N} M^{|\tau|-|\gamma|}\left\|P_{M} u\right\|_{L^{2}} M^{|\gamma|+1}\left\|P_{N} u\right\|_{L^{\infty}} \\
& \lesssim\left(\sum_{M} M^{2|\tau|}\left\|P_{M} u\right\|_{L^{2}}^{2}\right)^{\frac{1}{2}}\left(\sum_{M} M^{2}\left\|P_{M} u\right\|_{L^{\infty}}^{2}\right)^{\frac{1}{2}} \\
& \lesssim\|u\|_{H^{|\tau|} \mid}\|u\|_{W^{1+\delta, \infty}} .
\end{aligned}
$$

Independently, we estimate $T_{1}$ as follows using that if $16 M_{i} \leq N_{i}, i=1,2$ then

$$
\left\langle P_{M_{1}} f P_{N_{1}} g, P_{M_{2}} h P_{N_{2}} k\right\rangle_{L^{2} \times L^{2}}=0
$$

unless $N_{1} \leq 4 N_{2} \leq 16 N_{1}$ (intersection of the Fourier support), and letting $f=$ $D^{\tau-\gamma} u, g=D^{\gamma} \partial_{j} u$, we get

$$
\begin{aligned}
\left\|T_{1}\right\|_{L^{2}} & \lesssim \sum_{N_{1} \sim N_{2}, 16 M_{i} \leq N_{i}}\left\langle P_{N_{1}} f P_{M_{1}} g, P_{N_{2}} f P_{M_{2}} g\right\rangle_{L^{2} \times L^{2}} \\
& \lesssim\|u\|_{W^{1, \infty}}^{2} \sum_{N_{1} \sim N_{2}, 16 M_{i} \leq N_{i}}\left\|P_{N_{1}} f\right\|_{L^{2}}\left\|P_{N_{2}} f\right\|_{L^{2}}\left(M_{1} M_{2}\right)^{|\gamma|} \\
& \lesssim\|u\|_{W^{1, \infty}}^{2} \sum_{N_{1} \sim N_{2}} N_{1}^{|\gamma|}\left\|P_{N_{1}} f\right\|_{L^{2}} N_{2}^{|\gamma|}\left\|P_{N_{2}} f\right\|_{L^{2}} \\
& \lesssim\|u\|_{W^{1, \infty}}^{2}\|u\|_{H^{|\tau|}}^{2}
\end{aligned}
$$

and $T_{2}$ is treated exactly in the same way.

We also need the following "tame" product estimate (see e.g. Tao [25])

Lemma 1.2. For $1<p<\infty, s \geq 0$,

$$
\|u v\|_{W^{s, p}} \lesssim\|u\|_{L^{\infty}}\|v\|_{W^{s, p}}+\|u\|_{W^{s, p}}\|v\|_{L^{\infty}}
$$

for $u$ and $v$ in $L^{\infty} \cap W^{s, p}$.

\section{Linear Decay}

In this section, we investigate the decay of linear solutions of the linearized equation

$$
\partial_{t t} \rho-\Delta \rho-\Delta(-\Delta+1)^{-1} \rho=0 .
$$

These solutions can be expressed in terms of the initial data and of one "half-wave" operator

$$
T_{t}=e^{i t p(|\nabla|)}
$$

for $p$ defined in (1.6) that we now study. Our main result in this section is the following

Proposition 2.1. For any $\delta>0$, for any $f \in W^{\frac{5}{2}+\delta, 1}$, there holds that

$$
\left\|e^{i t p(|\nabla|)} f\right\|_{L^{\infty}} \lesssim \delta\left(|t|^{-\frac{4}{3}}+|t|^{-\frac{3}{2}}\right)\|f\|_{W^{\frac{5}{2}+\delta, 1}}
$$

for all $t \neq 0$. Besides, we have the $L^{10}$-decay estimate

$$
\left\|e^{i t p(|\nabla|)} f\right\|_{L^{10}} \lesssim(1+|t|)^{-\frac{16}{15}}\|f\|_{W^{\frac{12}{5}, \frac{10}{9}}}
$$

uniformly in $\varepsilon, t, f$. 
More precise estimates are derived below. The rest of the section in devoted to a proof of (2.2) and (2.3).

For most of this section, we study the dispersive features of our operator in general dimension $n$. Proposition 2.1 is a consequence of the particular case $n=3$. Direct computations give that

$$
\begin{aligned}
p^{\prime}(r) & =\frac{1}{\sqrt{\left(1+r^{2}\right)\left(2+r^{2}\right)}}\left(1+r^{2}+\frac{1}{1+r^{2}}\right), \\
p^{\prime \prime}(r) & =\frac{r\left(r^{4}-2 r^{2}-6\right)}{\left(1+r^{2}\right)\left[\left(1+r^{2}\right)\left(2+r^{2}\right)\right]^{\frac{3}{2}}} \text { and } \\
p^{\prime \prime \prime}(r) & =\frac{5 r^{4}-6 r^{2}-6}{\left(1+r^{2}\right)^{\frac{5}{2}}\left(2+r^{2}\right)^{\frac{3}{2}}}-\frac{r\left(r^{4}-2 r^{2}-6\right)\left(11 r^{3}+16 r\right)}{\left(1+r^{2}\right)^{\frac{7}{2}}\left(2+r^{2}\right)^{\frac{5}{2}}} .
\end{aligned}
$$

We note that $p^{\prime \prime}(r)$ has one unique positive root at

$$
r=r_{0}=\sqrt{1+\sqrt{7}}
$$

In order to state our first result, we define a frequency localization function around the critical point $r_{0}$. Let $\psi_{r_{0}} \in C^{\infty}(\mathbb{R})$ be a smooth function such that $0 \leq \psi \leq 1, \psi_{r_{0}}\left(r_{0}+r\right)=1$ when $|r| \leq \varepsilon$ and $\psi_{r_{0}}\left(r_{0}+r\right)=0$ when $|r| \geq 2 \varepsilon$.

Lemma 2.1. For all time $t \neq 0$, and all $f \in L^{1}$, there holds that

$$
\left\|e^{i t p(|\nabla|)} \psi_{r_{0}}(|\nabla|) f\right\|_{L^{\infty}} \lesssim n, \varepsilon(1+|t|)^{-\frac{n-1}{2}-\frac{1}{3}}\|f\|_{L^{1}} .
$$

Proof. We note that

$$
\begin{aligned}
\left\|e^{i t p(|\nabla|)} \psi_{r_{0}}(|\nabla|) f(x)\right\|_{\infty} & =\left\|\mathcal{F}^{-1}\left\{e^{i t p(|\xi|)} \psi_{r_{0}}(|\xi|) \hat{f}(\xi)\right\}\right\|_{\infty} \\
& =\left\|\mathcal{F}^{-1}\left\{e^{i t p(|\xi|)} \psi_{r_{0}}(|\xi|)\right\} * f(x)\right\|_{\infty} \\
& \leq\left\|\mathcal{F}^{-1}\left\{e^{i t p(|\xi|)} \psi_{r_{0}}(|\xi|)\right\}\right\|_{\infty}\|f\|_{L^{1}} .
\end{aligned}
$$

Since $\psi_{r_{0}}$ is chosen to be spherically symmetric, it is well-known that

$$
\begin{aligned}
\mathcal{F}^{-1}\left\{e^{i t p(|\xi|)} \psi_{r_{0}}(|\xi|)\right\}(x) & =2 \pi \int_{0}^{\infty} e^{i t p(r)} \psi_{r_{0}}(r) \tilde{J}_{\frac{n-2}{2}}(r|x|) r^{n-1} d r \\
& =2 \pi \int_{0}^{\infty} e^{i t p(r)} \psi_{r_{0}}(r) \tilde{J}_{\frac{n-2}{2}}(r|x|) r^{n-1} d r
\end{aligned}
$$

where for all $n \geq 2$,

$$
\tilde{J}_{\frac{n-2}{2}}(s) \equiv s^{-\frac{n-2}{2}} J_{\frac{n-2}{2}}(s)=\operatorname{Re}\left(e^{i s} Z(s)\right)=e^{i s} Z(s)-e^{-i s} \bar{Z}(s) .
$$

Here $Z(s)$ is a smooth function satisfying (cf John [14]) that for all $k \geq 0$ and all $s$

$$
\left|\partial^{k} Z(s)\right| \lesssim_{n, k}(1+s)^{-\frac{n-1}{2}-k} .
$$

We first estimate $e^{-i r|x|} \bar{Z}(r|x|)$. Changing variable $r \rightarrow r+r_{0}$, and letting $\Psi(v)=$ $\left(r_{0}+r\right)^{n-1} \psi_{r_{0}}\left(r_{0}+r\right)$, we get

$$
\begin{aligned}
\tilde{I}_{1} & =\int_{0}^{\infty} e^{i(t p(r)-r|x|)} \psi(r) \bar{Z}(r|x|) r^{n-1} d r \\
& =\int_{-2 \varepsilon}^{2 \varepsilon} e^{i(t p(r)-r|x|)} \Psi(r) \bar{Z}\left(\left(r_{0}+r\right)|x|\right) d r
\end{aligned}
$$


and a first crude estimate allow us to conclude that

$$
\left|\tilde{I}_{1}\right| \lesssim_{n, k, \varepsilon} 1
$$

which takes care of the small times $|t| \lesssim 1$. Thus, we now assume that $t>1$. We consider the phase

$$
\Omega(r,|x|, t)=\left(p(r)-r \frac{|x|}{t}\right) .
$$

By (2.4), we directly compute that $p^{\prime}\left(r_{0}\right) \neq 0$ and

$$
p^{\prime \prime \prime}\left(r_{0}\right)=\frac{4 r_{0}^{4}-4 r_{0}^{2}}{\left(1+r_{0}^{2}\right)^{\frac{5}{2}}\left(2+r_{0}^{2}\right)^{\frac{3}{2}}} \neq 0 .
$$

Case 1 Suppose that $|x| \geq \frac{1}{4} p^{\prime}\left(r_{0}\right) t$. Then, since $|r| \leq 2 \varepsilon$,

$$
\left|\partial_{r}^{3} \Omega(r,|x|, t)\right|=\left|p^{\prime \prime \prime}(r)\right|>\frac{1}{2}\left|p^{\prime \prime \prime}\left(r_{0}\right)\right|,
$$

if $\varepsilon>0$ is chosen sufficiently small, and using (2.8), by the Van der Corput lemma (see e.g. Stein [24]), we get that

$$
\begin{aligned}
\left|\tilde{I}_{1}\right| & \lesssim_{\varepsilon}|t|^{-\frac{1}{3}}\left(\sup _{k \in\{0,1\},|r| \leq 2 \varepsilon}\left|\bar{Z}\left(\left(r_{0}+r\right)|x|\right) \partial^{k} \Psi(r)\right|+|x| \sup _{|r| \leq 2 \varepsilon}\left|\Psi(r) \bar{Z}^{\prime}\left(\left(r_{0}+r\right)|x|\right)\right|\right) \\
& \lesssim_{\varepsilon}|t|^{-\frac{1}{3}}|x|^{-\frac{n-1}{2}} \\
& \lesssim_{\varepsilon}|t|^{-\frac{1}{3}-\frac{n-1}{2}}
\end{aligned}
$$

Case 2 Suppose now that $|x| \leq \frac{1}{4} p^{\prime}\left(r_{0}\right) t$. Then

$$
\left|\partial_{r} \Omega(r,|x|, t)\right|=\left|p^{\prime}\left(r_{0}\right)\right|-\frac{|x|}{t} \geq\left|p^{\prime}\left(r_{0}\right)\right| / 2
$$

and therefore, using the nonstationary phase and the fact that $Z$ has all derivatives bounded, we obtain that

$$
\left|\tilde{I}_{1}\right| \lesssim|t|^{-\frac{n}{2}} .
$$

The estimation of $e^{i r|x|} Z(r|x|)$ is easier. Proceeding as above, we introduce

$$
\tilde{I}_{2}=\int_{-2 \varepsilon}^{2 \varepsilon} e^{i(t p(r)+r|x|)} \Psi(r) Z\left(\left(r_{0}+r\right)|x|\right) d r .
$$

But the phase in $\tilde{I}_{2}$ satisfies

$$
\left|\partial_{r} \Omega_{2}(r,|x|, t)\right|=\left|\partial_{r}\left(p(r)+r \frac{|x|}{t}\right)\right| \geq\left|p^{\prime}(r)\right| \gtrsim 1
$$

and we can conclude as in Case 2 above to get

$$
\left|\tilde{I}_{2}\right| \lesssim|t|^{-\frac{n}{2}}
$$

Now this, (2.9), (2.10) and (2.11) prove (2.6).

Now that we have dealt with the degeneracy at $r_{0}$, the other degeneracy at 0 and $\infty$ are more easily dealt with at the price of loosing derivatives. To isolate these regions, we introduce two smooth cut-off functions. We let $\psi_{0}$ and $\psi_{\infty}$ such 
that $0 \leq \psi_{0}+\psi_{\infty} \leq 1, \psi_{0}$ is supported on $\left(-r_{0}+\varepsilon, r_{0}-\varepsilon\right), \psi_{\infty}$ is supported on $\left\{|x| \geq r_{0}+\varepsilon\right\}$ and

$$
\psi_{0}+\psi_{r_{0}}+\psi_{\infty}=1
$$

We first treat the case of small frequencies. We note that since $r_{0}$ is the only positive root of $p^{\prime \prime}, p^{\prime \prime}(r) \neq 0$ for either $r \in\left(r_{0}+\varepsilon, \infty\right)$ or $r \in\left(0, r_{0}-\varepsilon\right)$. Therefore we can apply Theorem 1 from Guo, Peng and Wang [11, case $(a)$ and (b) respectively, to obtain with (2.4):

Lemma 2.2. There holds that, for all $f \in L^{1}$

$$
\left\|e^{i t p(|\nabla|)} \psi_{0}(|\nabla|) f\right\|_{L^{\infty}} \lesssim n, \varepsilon(1+|t|)^{-\frac{n}{2}}\left\||\nabla|^{\frac{n-2}{2}} f\right\|_{L^{1}} .
$$

Lemma 2.3. For all $f \in L^{1}$, there holds that

$$
\left\|e^{i t p(|\nabla|)} \psi_{\infty}(|\nabla|) f\right\|_{L^{\infty}} \lesssim_{n, \varepsilon}|t|^{-\frac{n}{2}}\left\||\nabla|^{\frac{n+2}{2}} f\right\|_{B_{1,1}^{0}} .
$$

Finally, from Lemma 2.12.2 2.3] we can prove Proposition 2.1.

Proof of Proposition 2.1, (2.2) follows directly from (2.1), (2.13) and (2.14). In order to get (2.3), we interpolate between the isometric property

$$
\left\|e^{i t p(|\nabla|)} P f\right\|_{L^{2}}=\|P f\|_{L^{2}}
$$

for $P$ a Fourier projector and the various $L^{\infty}$ estimates. Interpolating with (2.1) gives that

$$
\left\|e^{i t p(|\nabla|)} \psi(|\nabla|) f\right\|_{L^{10}} \lesssim|t|^{-\frac{16}{15}}\|f\|_{L^{\frac{10}{9}}} .
$$

Interpolating with (2.13) gives

$$
\left\|e^{i t p(|\nabla|)} \psi_{0}(|\nabla|) f\right\|_{L^{10}} \lesssim|t|^{-\frac{6}{5}}\|f\|_{L^{\frac{10}{9}}} .
$$

Finally, interpolating with (2.14) and using the inclusions of Besov spaces

$$
L^{10} \subset B_{10,2}^{0} \text { and } B_{\frac{10}{9}, 2}^{0} \subset L^{\frac{10}{9}},
$$

and Bernstein estimates (1.11), we get that

$$
\begin{aligned}
\left\|e^{i t p(|\nabla|)} \psi_{\infty}(|\nabla|) f\right\|_{L^{10}}^{2} & \lesssim \sum_{N \geq 1}\left\|e^{i t p(|\nabla|)} \psi_{\infty}(|\nabla|) P_{N} f\right\|_{L^{10}}^{2} \\
& \lesssim|t|^{-\frac{12}{5}} \sum_{N \geq 1} N^{4}\left\|P_{N} f\right\|_{L^{\frac{10}{9}}}^{2} \\
& \lesssim|t|^{-\frac{12}{5}}\|f\|_{W^{2, \frac{10}{9}}}^{2}
\end{aligned}
$$

Since for small time $t \leq 1$, we also have that

$$
\left\|e^{i t p(|\nabla|)} f\right\|_{L^{10}} \lesssim\left\|e^{i t p(|\nabla|)} f\right\|_{H^{\frac{6}{5}}} \lesssim\|f\|_{H^{\frac{6}{5}}} \lesssim\|f\|_{W^{\frac{12}{5}}, \frac{10}{9}}
$$

and since $f=\psi_{r_{0}}(|\nabla|) f+\psi_{0}(|\nabla|) f+\psi_{\infty}(|\nabla|) f$, this ends the proof. 


\section{NORMAL FORM TRANSFORMATION}

In this section, we derive the normal form transformation for $\alpha_{j}$. Isolating linear, quadratic and higher order terms, we can rewrite the Euler-Poisson system (1.4) as follows:

$$
\begin{array}{lll}
\partial_{t} \rho+\operatorname{div}(v) & +\operatorname{div}(\rho v) & =0 \\
\partial_{t} v+\nabla \rho+\nabla \phi & +(v \cdot \nabla) v-\nabla \frac{\rho^{2}}{2} & =-\nabla\left[\ln (1+\rho)-\rho+\frac{\rho^{2}}{2}\right] \\
\rho=(1-\Delta) \phi & +\frac{\phi^{2}}{2} & +\left[e^{\phi}-1-\phi-\frac{\phi^{2}}{2}\right] .
\end{array}
$$

The last line defines an operator $\rho \mapsto \phi(\rho)$ such that

$$
\phi(\rho)=(1-\Delta)^{-1} \rho-\frac{1}{2}(1-\Delta)^{-1}\left[(1-\Delta)^{-1} \rho\right]^{2}+R(\rho)
$$

where $R$ satisfies good properties. We note that since $\nabla \times v=0$ there exists a function $\psi$ such that $v=\nabla \psi$ and consequently, $(v \cdot \nabla) v=\nabla \frac{|v|^{2}}{2}$. In terms of the velocity potential $\psi$, we can rewrite the above system as

$$
\begin{gathered}
\partial_{t}\left(\begin{array}{c}
\rho \\
\psi
\end{array}\right)+\left(\begin{array}{cc}
0 & \Delta \\
(1-\Delta)^{-1}+1 & 0
\end{array}\right)\left(\begin{array}{c}
\rho \\
\psi
\end{array}\right) \\
=\left(\begin{array}{c}
-\nabla \cdot(\rho \nabla \psi) \\
\frac{1}{2}(1-\Delta)^{-1}\left[(1-\Delta)^{-1} \rho\right]^{2}-R(\rho)-\ln (1+\rho)+\rho-\frac{\rho^{2}}{2}
\end{array}\right) .
\end{gathered}
$$

We denote the pair of eigenfunctions of the linear part as $\left(\begin{array}{c}1 \\ \pm \frac{p(|\nabla|)}{i|\nabla|^{2}}\end{array}\right)=\left(\begin{array}{c}1 \\ \pm \frac{q(|\nabla|)}{i|\nabla|}\end{array}\right)$, and recall $\alpha_{j}=\rho+\frac{(-1)^{j} i}{q(|\nabla|)} \mathcal{R}^{-1} v \equiv \rho+\frac{(-1)^{j} i}{q(|\nabla|)}|\nabla| \psi$, where $\mathcal{R}=\frac{\nabla}{|\nabla|}$ stands for the Riesz transform. We can diagonalize the matrix as:

$$
\left(\begin{array}{cc}
0 & \Delta \\
(1-\Delta)^{-1}+1 & 0
\end{array}\right)=\left(\begin{array}{cc}
1 & 1 \\
\frac{q(|\nabla|)}{i|\nabla|} & -\frac{q(|\nabla|)}{i|\nabla|}
\end{array}\right)\left(\begin{array}{cc}
i p(|\nabla|) & 0 \\
0 & -i p(|\nabla|)
\end{array}\right)\left(\begin{array}{cc}
\frac{1}{2} & \frac{i|\nabla|}{2 q(|\nabla|)} \\
\frac{1}{2} & -\frac{i|\nabla|}{2 q(|\nabla|)}
\end{array}\right)
$$

Now, with $\alpha$ given in (1.8), using that $\mathcal{R}^{-1} \nabla=|\nabla| \mathcal{R}^{-1} \frac{\nabla}{|\nabla|}=|\nabla|$, and

$$
\operatorname{div}(v)=\operatorname{div}\left(\frac{\nabla}{|\nabla|} \mathcal{R}^{-1} v\right)=-|\nabla| \mathcal{R}^{-1} v,
$$

we diagonalize the matrix and rewrite (3.5) in terms of $\alpha$ as

$$
\left(\partial_{t}+(-1)^{j} i p(|\nabla|)\right) \alpha_{j}=Q_{j}(\alpha)+\mathcal{N}_{j}
$$

where $Q_{2}=\bar{Q}_{1}$, and $\mathcal{N}_{2}=\overline{\mathcal{N}}_{1}$ such that the quadratic term $Q_{1}$ and the cubic term $\mathcal{N}_{1}$ take the form:

$$
\begin{aligned}
& Q_{j}=-\operatorname{div}(\rho v)+(-1)^{j} \frac{i|\nabla|}{2 q(|\nabla|)}\left\{(1-\Delta)^{-1}\left[(1-\Delta)^{-1} \rho\right]^{2}-\rho^{2}-|v|^{2}\right\} \\
& \mathcal{N}_{j}=(-1)^{j} \frac{i|\nabla|}{q(|\nabla|)}\left[\ln (1+\rho)-\rho+\frac{\rho^{2}}{2}-R(\rho)\right] .
\end{aligned}
$$

The most important step is to study the linear profiles

$$
\omega_{j}(t)=e^{(-1)^{j} i t p(|\nabla|)} \alpha_{j}(t),
$$


so that its temporal derivatives are of at least of quadratic order:

$$
\partial_{t} \omega_{j}=e^{(-1)^{j} i p(|\nabla|) t}\left\{Q_{j}(\alpha)+\mathcal{N}_{j}\right\} .
$$

Plugging $\rho=\frac{\alpha_{1}+\alpha_{2}}{2}$ and $v=\frac{\nabla p(|\nabla|)}{-\Delta} \frac{\alpha_{1}-\alpha_{2}}{2 i}$ into $Q_{j}$, we now compute the Fourier transform of $Q_{j}(\alpha)$ as

$$
\begin{aligned}
& \hat{Q}_{j}(\alpha)(t, \xi) \\
& =\int_{\mathbb{R}^{3}}\left[-\frac{1}{4} \frac{\xi \cdot \eta}{|\eta|} q(\eta) \hat{\alpha}_{1}(\xi-\eta) \hat{\alpha}_{1}(\eta)+\frac{1}{4} \frac{\xi \cdot \eta}{|\eta|} q(\eta) \hat{\alpha}_{2}(\xi-\eta) \hat{\alpha}_{2}(\eta)\right. \\
& +\frac{i|\xi|}{8 q(\xi)}\left(\frac{(\xi-\eta) \cdot \eta}{|\xi-\eta||\eta|} q(\xi-\eta) q(\eta)-1+\frac{1}{\langle\xi\rangle^{2}\langle\xi-\eta\rangle^{2}\langle\eta\rangle^{2}}\right) \hat{\alpha}_{1}(\xi-\eta) \hat{\alpha}_{2}(\eta) \\
& -\frac{(-1)^{j} i|\xi|}{8 q(\xi)}\left(\frac{(\xi-\eta) \cdot \eta}{|\xi-\eta||\eta|} q(\xi-\eta) q(\eta)-1+\frac{1}{\langle\xi\rangle^{2}\langle\xi-\eta\rangle^{2}\langle\eta\rangle^{2}}\right) \hat{\alpha}_{1}(\xi-\eta) \hat{\alpha}_{1}(\eta) \\
& +\frac{1}{4} \frac{\xi \cdot \eta}{|\eta|} q(\eta) \hat{\alpha}_{1}(\xi-\eta) \hat{\alpha}_{2}(\eta)-\frac{1}{4} \frac{\xi \cdot \eta}{|\eta|} q(\eta) \hat{\alpha}_{2}(\xi-\eta) \hat{\alpha}_{1}(\eta) \\
& \left.-\frac{(-1)^{j} i|\xi|}{8 q(\xi)}\left(\frac{(\xi-\eta) \cdot \eta}{|\xi-\eta||\eta|} q(\xi-\eta) q(\eta)-1+\frac{1}{\langle\xi\rangle^{2}\langle\xi-\eta\rangle^{2}\langle\eta\rangle^{2}}\right) \hat{\alpha}_{2}(\xi-\eta) \hat{\alpha}_{2}(\eta)\right] d \eta \\
& \equiv \int_{\mathbb{R}^{3}}\left[m_{r l}^{j}(\xi, \eta) \hat{\alpha}_{r}(\xi-\eta) \hat{\alpha}_{l}(\eta)\right](s) d s .
\end{aligned}
$$

We now integrate (3.6) to get

$$
\begin{aligned}
\hat{\alpha}_{j}(t) & =e^{(-1)^{j+1} i p(|\xi|) t} \hat{\alpha}_{j}(0)+\int_{0}^{t} e^{(-1)^{j+1} i p(|\xi|)(t-s)} \hat{Q}_{j}(\alpha)(s) d s \\
& +\int_{0}^{t} e^{(-1)^{j+1} i p(|\xi|)(t-s)} \hat{\mathcal{N}}_{1}(\alpha)(s) d s \\
& =e^{(-1)^{j+1} i p(|\xi|) t} \hat{\alpha}_{j}(0)+\int_{0}^{t} e^{(-1)^{j+1} i p(|\xi|)(t-s)} \hat{\mathcal{N}}_{j}(\alpha)(s) d s \\
& \left.+e^{(-1)^{j+1} i p(|\xi|) t} \int_{0}^{t} e^{(-1)^{j+1} i p(|\xi|) s} m_{r l}^{j} \hat{\alpha}_{r}(\xi-\eta) \hat{\alpha}_{l}(\eta)\right](s) d s(s) d s .
\end{aligned}
$$

The crucial step is to replace $\hat{\alpha}_{j}(s)=e^{(-1)^{j+1} i p(|\xi|) s} \hat{\omega}_{j}(s)$ in the third term, which then takes the form

$$
\hat{\Psi}_{j}(\alpha)=e^{(-1)^{j+1} i p(|\xi|) t} \sum_{r, l=1}^{2} \int_{0}^{t} \int_{\mathbb{R}^{3}} m_{r l}^{j} e^{i s \Phi_{r l}} \hat{\omega}_{r}(\xi-\eta) \hat{\omega}_{l}(\eta) d \eta d s .
$$

Here $\hat{\omega}_{1}(\xi)=e^{i t p(\xi)} \hat{\alpha}_{1}(\xi)$ and $\hat{\omega}_{2}(\xi)=e^{-i t p(\xi)} \hat{\alpha}_{2}(\xi)=\overline{\hat{\omega}}_{1}(\xi)$,

$$
\begin{aligned}
\Phi_{r l}(\xi, \eta) & =(-1)^{j+1} p(\xi)+(-1)^{r+1} p(\xi-\eta)+(-1)^{l+1} p(\eta), \text { and } \\
m_{r l}^{j}(\xi, \eta) & =|\xi| n_{1 r l}^{j}(\xi) n_{2 r l}^{j}(\xi-\eta) n_{3 r l}^{j}(\eta),
\end{aligned}
$$

is a factorable multiplier defined in (3.9), where the $n_{\text {olk }}^{j}$ are either smooth functions or product of a smooth function with the angle function $x \mapsto \frac{x}{|x|}$. More specifically, 
there are only four different phases of the following:

$$
\begin{aligned}
& \Phi_{1}(\xi, \xi-\eta, \eta)=p(\xi)-p(\xi-\eta)-p(\eta) \\
& \Phi_{2}(\xi, \xi-\eta, \eta)=p(\xi)+p(\xi-\eta)+p(\eta) \\
& \Phi_{3}(\xi, \xi-\eta, \eta)=p(\xi)-p(\xi-\eta)+p(\eta) \\
& \Phi_{4}(\xi, \xi-\eta, \eta)=p(\xi)+p(\xi-\eta)-p(\eta) .
\end{aligned}
$$

Integrating by parts in $s$ in the integral in $\Psi$, and making use of the fact that $\partial_{t} \hat{\omega}$ is at least quadratic by (3.8), we obtain from (3.11) that1]

$$
\begin{aligned}
& e^{(-1)^{j} i p(|\xi|) t} \hat{\Psi}_{j}(\alpha)(t, \xi) \\
& =\sum_{r, l=1}^{2}\left[\int_{\mathbb{R}^{3}} \frac{m_{r l}^{j}}{i \Phi_{r l}} e^{i s \Phi_{r l}} \hat{\omega}_{r}(\xi-\eta) \hat{\omega}_{l}(\eta) d \eta\right]_{s=0}^{t} \\
& +2 \sum_{r, l=1}^{2} \int_{0}^{t} \int_{\mathbb{R}^{3}} i \frac{m_{r l}^{j}(\xi, \eta)}{\Phi_{r l}} e^{i s \Phi_{r l} \hat{\omega}_{r}(\xi-\eta) \partial_{t} \hat{\omega}_{l}(\eta) d \eta d s} \\
& =i \sum_{r, l=1}^{2} \int_{\mathbb{R}^{3}} \frac{m_{r l}^{j}}{\Phi_{r l}} \hat{\omega}_{r}(0, \xi-\eta) \hat{\omega}_{l}(0, \eta) d \eta \\
& +\sum_{r, l=1}^{2} e^{(-1)^{j} i t p(\xi)} \int_{\mathbb{R}^{3}} \frac{m_{r l}^{j}}{i \Phi_{r l}} \hat{\alpha}_{r}(t, \xi-\eta) \hat{\alpha}_{l}(t, \eta) d \eta \\
& +2 \sum_{r, l, r_{1}, l_{1}=1}^{2} \int_{0}^{t} \int_{\mathbb{R}^{3}} \frac{i m_{r l}^{j}(\xi, \eta) m_{r_{1} l_{1}}^{l}(\eta, \zeta)}{\Phi_{r l}} e^{i s \Phi_{r l}} \hat{\omega}_{r}(\xi-\eta) e^{i s(-1)^{l} p(|\eta|)} \hat{Q}_{j}(\alpha)(\eta) d \eta d s \\
& +2 \sum_{r, l=1}^{2} \int_{0}^{t} \int_{\mathbb{R}^{3}} \frac{i m_{r l}^{j}}{\Phi_{r l}} e^{i s \Phi_{r l}} \hat{\omega}_{r}(\xi-\eta) e^{i s(-1)^{l} p(\eta)} \hat{\mathcal{N}}_{l}(\eta) d \eta d s
\end{aligned}
$$

We then change back to $\hat{\omega}_{j}(s)=e^{(-1)^{r+1} i p(|\xi|) s} \hat{\alpha}_{j}(s)$, and using (3.7), we write

$$
\begin{aligned}
& e^{(-1)^{j} i p(|\xi|) t}\left(\hat{\alpha}_{j}(t)+\mathfrak{B}_{j}(\alpha)\right) \\
= & \hat{\alpha}_{j}(0)+\mathfrak{B}_{j}(\alpha(0))+\int_{0}^{t} e^{(-1)^{j} i p(|\xi|) s} \hat{Q}_{j}(\alpha)(s) d s+\int_{0}^{t} e^{(-1)^{j} i p(|\xi|) s} \hat{\mathcal{N}}_{j}(\alpha)(s) d s \\
= & \hat{\alpha}_{j}(0)+\mathfrak{B}_{j}(\alpha(0))+\int_{0}^{t} e^{(-1)^{j} i p(|\xi|) s} \hat{\mathcal{N}}_{j}(\alpha)(s) d s \\
& +\int_{0}^{t} e^{(-1)^{j} i p(|\xi|) s} \frac{i m_{l k}^{j}(\xi, \eta)}{\Phi_{l k}} \hat{\alpha}_{r}(\xi-\eta) \hat{h}_{l}(\alpha(\eta))(s) d s(s) d s
\end{aligned}
$$

where the normal form transformation is

$$
\mathcal{F} \mathfrak{B}_{j}\left(\alpha_{j}\right)(\xi)=\sum_{r, l=1}^{2} \int_{\mathbb{R}^{3}} \frac{m_{r l}^{j}}{i \Phi_{r l}} \hat{\alpha}_{r}(\xi-\eta) \hat{\alpha}_{l}(\eta) d \eta
$$

\footnotetext{
${ }^{1}$ for notational simplicity, we do not distinguish $m_{l r}^{j}(\xi, \eta)$ and $m_{r l}^{j}(\xi, \xi-\eta)$.
} 
and

$$
\hat{h}_{l}(\alpha) \equiv \int_{\mathbb{R}^{3}} m_{r_{1} l_{1}}(\eta, \zeta) \hat{\alpha}_{r_{1}}(\eta-\zeta) \hat{\alpha}_{l_{1}}(\zeta) d \zeta+\hat{\mathcal{N}}_{l}
$$

is the associated cubic nonlinearity.

We next show that $h(\alpha)$ behaves like a quadratic term in $\alpha$.

Lemma 3.1. Assuming that $\alpha$ has small $X$-norm, then

$$
\left\||\nabla|^{-1} h(\alpha(t))\right\|_{H^{2 k}}+\left\||\nabla|^{-1} \mathcal{N}\right\|_{H^{2 k}} \lesssim(1+t)^{-\frac{16}{15}}\|\alpha\|_{X}^{2} .
$$

Proof. When $h$ is a product of $\alpha$ 's, this follows directly from the Sobolev embedding $L^{\infty} \subset W^{\frac{3}{10}, 10}$.

When $h=\mathcal{N}$, we see from (3.7) that, except for the term involving $R$, a similar proof works. For the terms involving $R$, we proceed as follows: Letting $E(x)=$ $e^{x}-1-x-\frac{x^{2}}{2}$, we see from (3.3). (3.4) that

$$
\begin{aligned}
& (1-\Delta) R+\frac{1}{2}\left[(1-\Delta)^{-1} \rho-(1-\Delta)^{-1}\left((1-\Delta)^{-1} \rho\right)^{2}\right] R+\frac{R^{2}}{2} \\
& +E\left((1-\Delta)^{-1} \rho-\frac{1}{2}(1-\Delta)^{-1}\left[(1-\Delta)^{-1} \rho\right]^{2}+R\right) \\
& =\frac{1}{2}(1-\Delta)^{-1} \rho\left[(1-\Delta)^{-1}\left[(1-\Delta)^{-1} \rho\right]^{2}\right]-\frac{1}{8}\left[(1-\Delta)^{-1}\left[(1-\Delta)^{-1} \rho\right]^{2}\right]^{2} .
\end{aligned}
$$

In order to solve (3.18), we define the following iterative scheme. For $\rho$ sufficiently small in $X$-norm, we let

$$
\begin{aligned}
& R_{0}=0 \\
& (1-\Delta) R_{k+1}=-\frac{1}{2}\left[(1-\Delta)^{-1} \rho-(1-\Delta)^{-1}\left((1-\Delta)^{-1} \rho\right)^{2}\right] R_{k}-\frac{R_{k}^{2}}{2} \\
& -E\left((1-\Delta)^{-1} \rho-\frac{1}{2}(1-\Delta)^{-1}\left[(1-\Delta)^{-1} \rho\right]^{2}+R_{k}\right) \\
& +\frac{1}{2}(1-\Delta)^{-1} \rho\left[(1-\Delta)^{-1}\left[(1-\Delta)^{-1} \rho\right]^{2}\right]-\frac{1}{8}\left[(1-\Delta)^{-1}\left[(1-\Delta)^{-1} \rho\right]^{2}\right]^{2}
\end{aligned}
$$

We see that, if $s>3 / 2$ and $p \geq 2$, using the tame estimate (1.12)

$$
\begin{aligned}
\left\|R_{k+1}\right\|_{W^{s+2, p}} & \lesssim\|\rho\|_{L^{\infty}}\left\|R_{k}\right\|_{W^{s, p}}+\|\rho\|_{W^{s-2, p}}\left\|R_{k}\right\|_{L^{\infty}}+\left\|R_{k}\right\|_{L^{\infty}}\left\|R_{k}\right\|_{W^{s, p}} \\
& +\|\rho\|_{L^{\infty}}\left(\|\rho\|_{W^{s-2, p}}^{2}+\|\rho\|_{W^{s-2, p}}^{3}\right) \\
& +C\left(\|\rho\|_{L^{\infty}}+\left\|R_{k}\right\|_{L^{\infty}}\right)\left(\left\|R_{k}\right\|_{W^{s, p}}+\|\rho\|_{W^{s-2, p}}\right)^{3}
\end{aligned}
$$

and, assuming that

$$
\sup _{k}\left\|R_{k}\right\|_{L^{\infty}}+\|\rho\|_{L^{\infty}} \leq 2
$$

we also see that

$$
\left\|R_{k+1}-R_{k}\right\|_{H^{2}} \lesssim\left(\|\rho\|_{L^{\infty}}+\sup _{k}\left\|R_{k}\right\|_{L^{\infty}}\right)\left\|R_{k}-R_{k-1}\right\|_{L^{2}} .
$$

Hence, if $\|\rho\|_{X}<1$ is sufficiently small, there holds that

$$
(1+t)^{\frac{16}{15}}\left\|R_{k}\right\|_{W^{s+2,10}}+\left\|R_{k}\right\|_{H^{2(s+1)}} \lesssim\|\rho\|_{X}^{3} \lesssim 1
$$


for all $0 \leq s \leq k$ and that $\left(R_{k}\right)_{k}$ is a Cauchy sequence in $H^{2}$, hence converges to a unique limit $R=R(\rho)$, the given function which solves (3.18) and satisfies

$$
(1+t)^{\frac{16}{15}}\|R(\rho)\|_{W^{k+2,10}}+\|R(\rho)\|_{H^{2(k+1)}} \lesssim\|\alpha\|_{X}^{3} .
$$

Using now that $W^{2,10} \subset L^{\infty}$, one recovers from (3.19) that for all $k$,

$$
(1+t)^{\frac{16}{15}}\left\|R_{k}(t)\right\|_{H^{2 k+2}} \lesssim\|\alpha\|_{X}^{3} .
$$

Passing to the limit in $k$, we finish the proof of (3.17).

\section{THE $L^{2}$-TYPE NORM}

In this section, we get control on the first part of the $X$-norm, namely, we control the $L^{2}$-based norms as follows

Proposition 4.1. Let $\alpha$ correspond to a solution of (1.4) by (1.8), then if $\alpha$ has small $X$-norm there holds that

$$
\|\alpha\|_{H^{-1} \cap H^{2 k}} \lesssim\|\alpha(0)\|_{Y}+\|\alpha\|_{X}^{\frac{3}{2}} .
$$

The remaining of this section is devoted to the proof of Proposition 4.1. We first control the high derivatives and then the $H^{-1}$-norm.

4.1. The Energy estimate. In this subsection, we use energy methods to control high derivatives of the solution in $L^{2}$, assuming a control on the $X$-norm, and most notably integrability of the solution in $L^{10}$-norms.

In order to prove this, we rewrite (1.4) into the symmetrized form

$$
\partial_{t} u+A_{j}(u) \partial_{j} u=(0,-\nabla \phi)
$$

where $u=\left(\ln (1+\rho), v_{1}, v_{2}, v_{3}\right)$,

$$
A_{j}=\left(\begin{array}{cc}
v_{j} & e_{j}^{T} \\
e_{j} & v_{j} I_{3}
\end{array}\right) .
$$

Now, for a multi-index $\tau$, we derive (4.1) $\tau$ times and take the scalar product with $D^{\tau} u$ to get

$$
\begin{aligned}
\frac{1}{2} \frac{d}{d t}\left\|D^{\tau} u\right\|_{L^{2}}^{2} & =-\left(A_{j}(u) D^{\tau} \partial_{j} u, D^{\tau} u\right)_{L^{2} \times L^{2}} \\
& -\sum_{\gamma<\tau} c_{\gamma}\left(D^{\tau-\gamma}\left[A_{j}(u)\right] D^{\gamma} \partial_{j}(u), D^{\tau} u\right)-\left(\nabla D^{\tau} \phi, D^{\tau} v\right)_{L^{2} \times L^{2}} \\
& \lesssim\|\operatorname{div}(v)\|_{L^{\infty}}\left\|D^{\tau} u\right\|_{L^{2}}^{2}+\sum_{\gamma<\tau} c_{\gamma}\left|\left(D^{\tau-\gamma}\left[A_{j}(u)\right] D^{\gamma} \partial_{j}(u), D^{\tau} u\right)_{L^{2} \times L^{2}}\right| \\
& +\left|\left(D^{\tau} \phi, D^{\tau} \operatorname{div}(v)\right)_{L^{2} \times L^{2}}\right| .
\end{aligned}
$$

Besides, using (3.1) and (3.4), one sees that

$$
\begin{aligned}
\left(D^{\tau} \phi, D^{\tau} \operatorname{div}(v)\right) & =\left(D^{\tau}(1-\Delta)^{-1} \rho, D^{\tau} \operatorname{div}(v)\right)-\left(\nabla D^{\tau} \tilde{R}(\rho), D^{\tau} v\right) \\
& =-\left(D^{\tau}(1-\Delta)^{-1} \rho, D^{\tau} \partial_{t} \rho\right)-\left(D^{\tau}(1-\Delta)^{-1} \rho, D^{\tau} \operatorname{div}(\rho v)\right) \\
& -\left(\nabla D^{\tau} \tilde{R}(\rho), D^{\tau} v\right)
\end{aligned}
$$

with

$$
\tilde{R}(\rho)=\frac{1}{2}(1-\Delta)^{-1}\left[(1-\Delta)^{-1} \rho\right]^{2}-R(\rho)
$$


and $R$ given in (3.4). Now, using Lemma 1.1. we remark that for all $\gamma<\tau$, there holds that

$$
\left\|D^{\tau-\gamma} u D^{\gamma} \partial_{j} u\right\|_{L^{2}} \lesssim\|u\|_{W^{2,10}}\|u\|_{H^{|\tau|}}
$$

and combining this with (3.20), we obtain

$$
\begin{aligned}
\frac{1}{2} \frac{d}{d t}\left(\left\|D^{\tau} u\right\|_{L^{2}}^{2}+\left\|(1-\Delta)^{-\frac{1}{2}} D^{\tau} \rho\right\|_{L^{2}}^{2}\right) & \lesssim\|u\|_{W^{2,10}}^{2}\|u\|_{H^{|\tau|} \mid}+\|R\|_{H^{|\tau|+1}}\|u\|_{H^{|\tau|}} \\
& \lesssim(1+t)^{-\frac{16}{15}}\|u\|_{X}^{3}
\end{aligned}
$$

as long as $\tau \leq 2 k$ and that $\|\alpha\|_{X}$ is sufficiently small. Finally, integrating this in time and remarking that

$$
\rho=\operatorname{Re}(\alpha) \text { and } v=q(|\nabla|) \mathcal{R} \operatorname{Im}(\alpha),
$$

we obtain that

$$
\|u\|_{H^{\tau}}^{2} \lesssim\|u(0)\|_{H^{\tau}}^{2}+\|u\|_{X}^{3}
$$

provided that $\tau \leq 2 k$. Since control of $\ln (1+\rho)$ in $L_{t}^{\infty} H_{x}^{\tau}$-norm gives control of $\rho$ in $L_{t}^{\infty} H_{x}^{\tau}$-norm, this gives us the global bound on the derivatives we needed.

4.2. The $H^{-1}$-norm. In this section, we control the $H^{-1}$ norm of the solution, which the other $L^{2}$ component of the component of the $X$-norm. We use (3.10) and we first deal with the quadratic terms $Q_{j}(\alpha)$, whose contribution can be written as a finite sum of terms like (recall that $\alpha_{1}=\bar{\alpha}_{2}$ )

$$
I=\mathcal{F}^{-1} \int_{0}^{t} e^{i(t-s) p(\xi)}|\xi| \frac{m}{|\xi|} \hat{\alpha}(\xi-\eta) \hat{\alpha}(\eta) d \eta
$$

where, from (3.12) we see that one can write

$$
m=|\xi| n_{1}(\xi) n_{2}(\xi-\eta) n_{3}(\eta)
$$

with $n_{i}(\zeta)=\frac{\zeta}{|\zeta|} \tilde{n}(\zeta)$ or $n_{i}(\zeta)=\tilde{n}(\zeta)$ for $\tilde{n}$ an $S^{0}$-symbol. In particular,

$$
\left\|n_{i}(|\nabla|) f\right\|_{L^{r}} \lesssim\|f\|_{L^{r}}
$$

for $1<r<\infty$. We use a standard energy estimate and the inclusion $L^{\infty} \subset W^{1,10}$ to get

$$
\begin{aligned}
\left\|\frac{I}{|\xi|}\right\|_{L^{2}} & \lesssim \int_{0}^{t}\left\|\int_{\mathbb{R}^{3}} \frac{m}{|\xi|} \hat{\alpha}(\xi-\eta) \hat{\alpha}(\eta) d \eta d s\right\|_{L^{2}} d s \\
& \lesssim \int_{0}^{t}\left\|\int_{\mathbb{R}^{3}}\left(n_{2}(\xi-\eta) \hat{\alpha}(\xi-\eta)\right)\left(n_{3}(\eta) \hat{\alpha}(\eta)\right) d \eta\right\|_{L^{2}} d s \\
& \lesssim \int_{0}^{t}\left\|\left(n_{2}(|\nabla|) \alpha\right)\left(n_{3}(|\nabla|) \alpha\right)\right\|_{L^{2}} d s \\
& \lesssim \int_{0}^{t}\left\|n_{2}(|\nabla|) \alpha\right\|_{L_{t}^{\infty} L_{x}^{2}}\left\|n_{3}(|\nabla|) \alpha(s)\right\|_{L_{x}^{\infty}} d s \\
& \lesssim\|\alpha\|_{X} \int_{0}^{t}\left\|(1-\Delta)^{\frac{1}{2}} n_{3}(|\nabla|) \alpha(s)\right\|_{L_{x}^{10}} d s \\
& \lesssim\|\alpha\|_{X} \int_{0}^{t}\left\|(1-\Delta)^{\frac{1}{2}} \alpha(s)\right\|_{L_{x}^{10}} \lesssim\|\alpha\|_{X}^{2} \int_{0}^{t} \frac{d s}{(1+s)^{\frac{16}{15}}} \\
& \lesssim\|\alpha\|_{X}^{2} .
\end{aligned}
$$


Next we control the contribution of the cubic term $\mathcal{N}$ as follows using the fact that $e^{i t p(|\nabla|)}$ is a unitary operator and (3.17),

$$
\begin{aligned}
\left\||\nabla|^{-1} \int_{0}^{t} e^{-i(t-s) p(|\nabla|)} \mathcal{N}(s) d s\right\|_{L^{2}} & \lesssim \int_{0}^{t}\left\||\nabla|^{-1} \mathcal{N}\right\|_{L^{2}} d s \\
& \lesssim\|\alpha\|_{X}^{2} \int_{0}^{t} \frac{d s}{(1+s)^{\frac{16}{15}}} \lesssim\|\alpha\|_{X}^{2} .
\end{aligned}
$$

Combining the two above estimates give that

$$
\begin{aligned}
\left\||\nabla|^{-1} \alpha\right\|_{L_{t}^{\infty} L_{x}^{2}} & \lesssim\left\||\nabla|^{-1} \alpha_{0}\right\|_{L^{2}}+\left\|\frac{I(\xi)}{|\xi|}\right\|_{L^{2}}+\left\|\int_{0}^{t} \frac{e^{-i(t-s) p(|\xi|)}}{|\xi|} \hat{\mathcal{N}}(s) d s\right\|_{L^{2}} \\
& \lesssim\left\|\alpha_{0}\right\|_{Y}+\|\alpha\|_{X}^{2}
\end{aligned}
$$

so that we control the first part in the $X$-norm.

\section{Bilinear Multiplier Theorem}

5.1. A general multiplier theorem. In order to control the last part of the norm, we need to deal with bilinear terms in (3.15), (3.16) which involve convolution with a singular symbol. Note that since $p(0)=0$, the symbol is quite singular on the whole parameter space and especially near $(\xi, \eta)=(0,0)$. In particular, we cannot use the traditional Coifman-Meyer multiplier theorem [2], or a more refined version as in Muscalu, Pipher, Tao and Thiele [18, 19] since in all these cases, the multiplier need to satisfy some homogeneity conditions. In order to overcome this we use estimates inspired from Gustafson, Nakanishi and Tsai 13 that we present now. Although most of the results in this subsection are essentially contained in Gustafson, Nakanishi and Tsai [13, for selfcontainness, we give a direct proof.

We introduce the following multiplier norm:

$$
\|\mathfrak{m}\|_{M_{\xi, \eta}^{s, b}}=\sum_{N \in 2^{\mathbb{Z}}}\left\|P_{N}^{\eta} \mathfrak{m}(\xi, \eta)\right\|_{L_{\xi}^{b} \dot{H}_{\eta}^{s}}
$$

and we let $\mathcal{M}_{\xi, \eta}^{s}=\mathcal{M}_{\xi, \eta}^{s, \infty}$, which will be the norm that we mostly use. To a multiplier $\mathfrak{m}$, we associate the bilinear "pseudo-product" operator

$$
B[f, g]=\mathcal{F}_{\xi}^{-1} \int_{\mathbb{R}^{3}} \mathfrak{m}(\xi, \eta) \hat{f}(\xi-\eta) \hat{g}(\eta) d \eta .
$$

Our goal in this section is to obtain robust estimates on $B$.

Lemma 5.1. If $\|\mathfrak{m}\|_{L_{\xi}^{\infty} \dot{H}_{\eta}^{s-\varepsilon}}+\|\mathfrak{m}\|_{L_{\xi}^{\infty} \dot{H}_{\eta}^{s+\varepsilon}}<\infty$, then the $M_{\xi, \eta}^{s}$-norm of $\mathfrak{m}$ is finite.

Proof. Indeed, we have that

$$
\begin{aligned}
\left\|P_{N}^{\eta} \mathfrak{m}(\xi, \eta)\right\|_{L_{\xi}^{\infty} \dot{H}_{\eta}^{s}} & \leq \min \left(N^{-\varepsilon}\|\mathfrak{m}\|_{L_{\xi}^{\infty} \dot{H}_{\eta}^{s+\varepsilon}}, N^{\varepsilon}\|\mathfrak{m}\|_{L_{\xi}^{\infty} \dot{H}_{\eta}^{s-\varepsilon}}\right), \text { so that } \\
\sum_{N}\left\|P_{N}^{\eta} \mathfrak{m}(\xi, \eta)\right\|_{L_{\xi}^{\infty} \dot{H}_{\eta}^{s}} & \lesssim\left(\sum_{N \leq 1}+\sum_{N \geq 1}\right)\left\|P_{N}^{\eta} \mathfrak{m}(\xi, \eta)\right\|_{L_{\xi}^{\infty} \dot{H}_{\eta}^{s}} \\
& \lesssim \sum_{N \leq 1} N^{\varepsilon}\|\mathfrak{m}\|_{L_{\xi}^{\infty} \dot{H}_{\eta}^{s-\varepsilon}}+\sum_{N \geq 1} N^{-\varepsilon}\|\mathfrak{m}\|_{L_{\xi}^{\infty} \dot{H}_{\eta}^{s+\varepsilon}}<+\infty .
\end{aligned}
$$


Theorem 5.1. Suppose that $0 \leq s \leq n / 2$ and $\|\mathfrak{m}\|_{M_{\eta, \xi}^{s, \infty}}=\|\mathfrak{m}\|_{M_{\eta, \xi}^{s}}<\infty$, then

$$
\|B[f, g]\|_{L^{l_{1}^{\prime}}} \lesssim\|\mathfrak{m}\|_{M_{\eta, \xi}^{s}}\|f\|_{L^{l_{2}}}\|g\|_{L^{2}}
$$

for $l_{1}, l_{2}$ satisfying

$$
2 \leq l_{1}, l_{2} \leq \frac{2 n}{n-2 s} \text { and } \frac{1}{l_{1}}+\frac{1}{l_{2}}=1-\frac{s}{n} .
$$

Remark 5.1. Actually, by changing coordinates $(\xi, \eta)$ to $(\xi, \zeta=\xi-\eta)$, we could replace the norm $M_{\xi, \eta}^{s}$ by

$$
\min \left(\|\mathfrak{m}\|_{M_{\xi, \eta}^{s}},\|\mathfrak{m}\|_{M_{\xi, \zeta}^{s}}\right) .
$$

Theorem 5.1 follows by duality from the following estimate which is an adaptation of an estimate from Gustafson, Nakanishi and Tsai [13].

Lemma 5.2. Let $0 \leq s \leq n / 2,2 \leq l_{1}, l_{2}, l_{3} \leq \frac{2 n}{n-2 s}$, then

$$
\|B[f, g]\|_{L^{l_{1}}} \lesssim\|\mathfrak{m}\|_{\mathcal{M}_{\xi, \eta}^{s, b}}\|f\|_{L^{l_{2}}}\|g\|_{L^{l_{3}}}
$$

for all $f \in L^{l_{2}}, g \in L^{l_{3}}$, where $\frac{1}{b}+\frac{1}{l_{1}}=\frac{1}{2}, \frac{1}{l_{2}}+\frac{1}{l_{3}}=1-\frac{s}{n}$.

Proof. We consider $\mathfrak{m}$ with finite $\mathcal{M}_{\xi, \eta}^{s, b}$ norm. Let $\mathcal{F}_{x}^{\eta}$ denote the Fourier transform from $x \rightarrow \eta$. By definition, we have

$$
\hat{f}(\eta) \hat{g}(\xi-\eta)=\mathcal{F}_{x}^{\eta} \mathcal{F}_{y}^{\xi} f(x+y) g(y)
$$

and we let $\mathfrak{m}_{N}(\xi, \eta)=P_{N}^{\eta} \mathfrak{m}(\xi, \eta)$ so that $\mathcal{F}_{z}^{\eta} \mathfrak{m}_{N}(\xi, \eta)=\chi\left(\frac{z}{N}\right) \mathcal{F}_{\eta}^{z} \mathfrak{m}_{N}(\xi, \eta)$. Using first Parseval's equality in $x$, then in $\eta$ and then in $\xi$, we see that

$$
\begin{aligned}
\int_{\mathbb{R}^{n}} B[f, g](x) h(x) d x & =\int_{\mathbb{R}^{2 n}} \mathfrak{m}_{N}(\xi, \eta) \hat{h}(\xi) \hat{f}(\eta) \hat{g}(\xi-\eta) d \eta d \xi \\
& =\int_{\mathbb{R}^{n}} \hat{h}(\xi) \int_{\mathbb{R}^{n}} \mathfrak{m}_{N}(\xi, \eta)\left(\mathcal{F}_{x}^{\eta} \mathcal{F}_{y}^{\xi} f(x+y) g(y)\right) d \eta d \xi \\
& =\int_{\mathbb{R}^{n}} \hat{h}(\xi) \int_{\mathbb{R}^{n}} \mathcal{F}_{x}^{\eta} \mathfrak{m}_{N}(\xi, \eta)\left(\mathcal{F}_{y}^{\xi} f(x+y) g(y)\right) d \eta d \xi \\
& =\int_{\mathbb{R}^{n}} \hat{h}(\xi) \int_{\mathbb{R}^{n}}\left(\chi\left(\frac{x}{N}\right) \mathcal{F}_{\eta}^{x} \mathfrak{m}_{N}(\xi, \eta)\right)\left(\mathcal{F}_{y}^{\xi} f(x+y) g(y)\right) d x d \xi \\
& =\int_{\mathbb{R}^{n}} \hat{h}(\xi) \int_{\mathbb{R}^{n}}\left(\mathcal{F}_{\eta}^{x} \mathfrak{m}_{N}(\xi, \eta)\right) \mathcal{F}_{y}^{\xi}\left(\chi\left(\frac{x}{N}\right) f(x+y) g(y)\right) d x d \xi
\end{aligned}
$$

We then use Cauchy-Schwarz's inequality for the inner integral for $x$, and then use the Hölder inequality with $\frac{1}{a}+\frac{1}{b}=\frac{1}{2}$ to get

$$
\begin{aligned}
& \int_{\mathbb{R}^{n}}|\hat{h}(\xi)|\left\|\mathcal{F}_{\eta}^{x} \mathfrak{m}_{N}(\xi, \eta)\right\|_{L_{x}^{2}}(\xi)\left\|\mathcal{F}_{y}^{\xi}\left\{\chi\left(\frac{x}{N}\right)(f(x+y) g(y))\right\}\right\|_{L_{x}^{2}}(\xi) d \xi \\
\leq & \|\hat{h}\|_{L_{\xi}^{a}}\left\|\mathfrak{m}_{N}(\xi, \eta)\right\|_{L_{\xi}^{b}\left(L_{\eta}^{2}\right)}\left\|\mathcal{F}_{y}^{\xi}\left\{\chi\left(\frac{x}{N}\right)(f(x+y) g(y))\right\}\right\|_{L_{x, \xi}^{2}} \\
\leq & \|h\|_{L_{x}^{a^{\prime}}}\left\|\mathfrak{m}_{N}(\xi, \eta)\right\|_{L_{\xi}^{b}\left(L_{\eta}^{2}\right)}\left\|\chi\left(\frac{x}{N}\right) f(x+y) g(y)\right\|_{L_{x, y}^{2}},
\end{aligned}
$$

where we have used the Hausdroff-Young's inequality for $a>2$, and the Parseval's equality in $\eta$ for the second factor, as well as the Parseval's equality in $\xi$ for the 
third factor. Finally, since $\left\|\chi\left(\frac{x}{N}\right)\right\|_{L^{n / s}} \lesssim N^{s}$, we employ the Hardy-Littlewood Young's inequality with $\frac{s}{n}+\frac{1}{l_{2}}+\frac{1}{l_{3}}=1$ to get that

$$
\left\|\chi\left(\frac{x}{N}\right) f(x+y) g(y)\right\|_{L_{x, y}^{2}} \lesssim N^{s}\|f\|_{L^{l_{1}}}\|g\|_{L^{l_{2}}} .
$$

Combining $N^{s}$ with $\|\mathfrak{m}(\xi, \eta)\|_{L_{\xi}^{b}\left(L_{\eta}^{2}\right)}$ with (5.4), we complete the proof.

In order to prove theorem [5.1, it suffices to remark that

$$
\begin{aligned}
\int_{\mathbb{R}^{n}} B[f, g](x) h(x) d x & =\int_{\mathbb{R}^{n}} \mathcal{F}_{x}^{\xi} B[f, g](\xi) \hat{h}(\xi) d \xi \\
& =\int_{\mathbb{R}^{2 n}} \hat{f}(\eta) \mathfrak{m}(\xi, \eta) \hat{h}(\xi) \hat{g}(\xi-\eta) d \xi d \eta \\
& =\int_{\mathbb{R}^{n}} f(x) B^{*}[h, \bar{g}](x) d x .
\end{aligned}
$$

Applying (5.5) to $B^{*}$ with $l_{1}=2$ to the bilinear operator corresponding to the multiplier $\mathfrak{m}^{*}(\xi, \eta)=\mathfrak{m}(\eta, \xi)$, we get the Theorem.

5.2. Multiplier Analysis. The control of $L^{10}$ norm is the main mathematical difficulty in this paper. In this subsection, we prove the relevant estimate to apply Theorem 5.1 to the multipliers that appear in our analysis.

Lemma 5.3. Let $a=b+c \in \mathbb{R}^{3}$, and let $|c| \leq \min \{|a|,|b|\}$, then

$$
|p(a)-p(b)-p(c)| \gtrsim|c|\{1-\cos [c, a]+1-\cos [b, a]\}+\frac{|a||b||c|}{(1+|a||b|)\left(1+|c|^{2}\right)} .
$$

where $[\cdot, \cdot]$ denote the angle between two vectors.

Proof. We first note that if $|b| \geq|a|$, then $p(b) \geq p(a)$ and

$$
|p(a)-p(b)-p(c)| \geq p(c) \gtrsim|c|
$$

and the lemma follows. We assume $|b| \leq|a|$. We remark that, as written in (1.6), $p(r)=r q(r)$, where $1 \leq q(r) \leq q(0)=\sqrt{2}$ and

$$
\begin{aligned}
& q^{\prime}(r)=-\frac{r}{\left(1+r^{2}\right)^{2} \sqrt{\frac{2+r^{2}}{1+r^{2}}}} \sim_{r \rightarrow \infty}-\frac{1}{r^{3}} \\
& q^{\prime}(0)=0, \quad q^{\prime \prime}(0)=-\frac{1}{\sqrt{2}} .
\end{aligned}
$$

From this, we get that

$$
p(a)-p(b)-p(c) \leq[|a|-|b|-|c|] q(a)-|b|(q(b)-q(a))-|c|(q(c)-q(a)) .
$$

From (5.8), we see that $q$ is decreasing and hence each term is non positive. Remarking that $|a|=|b| \cos [b, a]+|c| \cos [c, a]$, the first term above gives the first term on the right hand side in (5.7).

We now consider the last term in the right hand side. Notice first that if $\cos [c, a] \leq 9 / 10$, then the last term is bounded by $|c|(1-\cos [c, a])$ and the lemma is clearly valid. So we can assume that $c$ and $a$ are almost collinear with $\cos [c, a] \geq$ 9/10. In which case, we get that $|a| \geq 4 / 3|c|$ and

$$
|a|-|c| \sim|b| \sim|a| .
$$


Using (5.8), we see that there exists $\delta>0$ such that $-s \leq q^{\prime}(s) \leq-\frac{s}{2}$ for $0 \leq s \leq \delta$. Consequently, if $|a| \leq \delta$, we get that

$$
|c|(q(a)-q(c))=|c| \int_{|c|}^{|a|} q^{\prime}(s) d s \leq-|c| \frac{|a|^{2}-|c|^{2}}{4} \lesssim-|a||c|(|a|-|c|) \lesssim-|a||b||c| .
$$

On the other hand, since $q^{\prime}(r) \sim-r^{-3}$ at $\infty$, we see that

$$
q(a)-q(c)=\int_{|c|}^{|a|} q^{\prime}(s) d s \sim_{\infty}-\int_{|c|}^{|a|} \frac{d s}{s^{3}}=\frac{|c|^{2}-|a|^{2}}{2|a|^{2}|c|^{2}} \lesssim-\frac{|a|-|c|}{|a||c|^{2}}
$$

so that if $|c| \geq \delta^{-1}$ is sufficiently large, we get that

$$
|c|(q(a)-q(c)) \lesssim-\frac{1}{|c|} .
$$

Finally, in the last case $\delta \leq|a| \leq \delta^{-1}$ and $|a|=|c|+(|a|-|c|) \geq|c|+\delta / 2$. Therefore,

$$
\int_{|c|}^{|a|} q^{\prime}(s) d s \lesssim \int_{|c|}^{|c|+\delta / 2} q^{\prime}(s) d s \lesssim-\frac{\delta}{2} q^{\prime}\left(2 \delta^{-1}\right)
$$

and we recover the last term once again.

In the remaining part of this section, we consider the triangle with vertices $\xi, \eta, \xi-\eta$ and let $\theta$ be the angle between $\xi$ and $\eta(0 \leq \theta \leq \pi), \gamma$ the angle between $\xi$ and $\xi-\eta(0 \leq \gamma \leq \pi)$ and we let the angle between $\eta$ and $\eta-\xi$ by $\pi-\beta$ such that $\beta=\gamma+\theta$. We note that $\sin \frac{\beta}{2} \leq \frac{\beta}{2}$ and $\sin \frac{\beta}{2} \backsim \beta$ for $0 \leq \beta \leq \pi$ so that

$$
1-\cos \beta=2 \sin ^{2} \frac{\beta}{2} \backsim \beta^{2} .
$$

We now obtain general bounds on the multipliers that arise in our analysis. We first focus on the multiplier associated with the phase $\Phi_{1}$. In the end, in Section 6, we recover the bounds on the other multipliers using symmetry.

Lemma 5.4. The following estimates on $\Phi_{1}$ are globally true:

$$
\begin{aligned}
\left|\partial_{\xi} \Phi_{1}(\xi, \eta)\right| & \lesssim \frac{|\eta|}{\langle\max \{|\xi-\eta|,|\xi|\}\rangle\langle\min \{|\xi-\eta|,|\xi|\}\rangle^{2}}+|\sin \gamma| \\
\left|\partial_{\eta} \Phi_{1}(\xi, \eta)\right| & \lesssim \frac{|\xi|}{\langle\max \{|\xi-\eta|,|\eta|\}\rangle\langle\min \{|\xi-\eta|,|\eta|\}\rangle^{2}}+|\sin \beta| \\
\left|\Delta_{\xi} \Phi_{1}(\xi, \eta)\right| & \lesssim \frac{|\eta|}{\langle\max \{|\xi-\eta|,|\xi|\}\rangle\langle\min \{|\xi-\eta|,|\xi|\}\rangle^{3}}+\frac{|\eta|}{|\xi-\eta||\xi|} \\
\left|\Delta_{\eta} \Phi_{1}(\xi, \eta)\right| & \lesssim \frac{1}{\min (|\xi-\eta|,|\eta|)}
\end{aligned}
$$

for all $\xi, \eta \in \mathbb{R}^{3}$. 
Proof. Recall $\Phi_{1}=p(\xi)-p(\xi-\eta)-p(\eta)$. We compute

$$
\begin{aligned}
\left|\nabla_{\xi} \Phi_{1}\right| & =\left|p^{\prime}(\xi) \frac{\xi}{|\xi|}-p^{\prime}(\xi-\eta) \frac{\xi-\eta}{|\xi-\eta|}\right| \\
& \leq\left|p^{\prime}(\xi)-p^{\prime}(\xi-\eta)\right|+\left|p^{\prime}(\xi)\right| \frac{\xi}{|\xi|}-\frac{\xi-\eta}{|\xi-\eta|} \mid \\
& \lesssim\left|\int_{|\xi-\eta|}^{|\xi|} p^{\prime \prime}(s) d s\right|+\left|\frac{\xi}{|\xi|}-\frac{\xi-\eta}{|\xi-\eta|}\right| \\
& \lesssim\left|\int_{|\xi-\eta|}^{|\xi|} p^{\prime \prime}(s) d s\right|+2 \sin \frac{\gamma}{2} .
\end{aligned}
$$

We claim that

$$
\left|p^{\prime}(\xi)-p^{\prime}(\xi-\eta)\right| \lesssim \frac{|\eta|}{\langle\max \{|\xi-\eta|,|\xi|\}\rangle\langle\min \{|\xi-\eta|,|\xi|\}\rangle^{2}}
$$

In fact, if $\max (|\xi|,|\xi-\eta|) \leq 20$, from (2.4), using the crude bound $\left|p^{\prime \prime}(s)\right| \lesssim 1$, we obtain that

$$
\left|\int_{|\xi-\eta|}^{|\xi|} p^{\prime \prime}(s) d s\right| \lesssim|| \xi|-| \xi-\eta|| \lesssim|\eta| \lesssim \frac{|\eta|}{\langle\max \{|\xi-\eta|,|\xi|\}\rangle\langle\min \{|\xi-\eta|,|\xi|\}\rangle^{2}}
$$

Therefore, we only need to consider the case $\max \{|\xi|,|\xi-\eta|\} \geq 20$. Then, if $\min \{|\xi|,|\xi-\eta|\} \leq 10$, we get that $|\eta| \simeq \max \{|\xi|,|\xi-\eta|\}$ and the right-hand side of (5.10) is of order 1 and the claim is valid. Finally, if $\min \{|\xi|,|\xi-\eta|\} \geq 10$, from (2.4), $p^{\prime \prime}(r) \sim \frac{1}{r^{3}}$ as $r \rightarrow \infty$, and we conclude that claim since

$$
\begin{aligned}
\left|\int_{|\xi-\eta|}^{|\xi|} p^{\prime \prime}(s) d s\right| & \lesssim\left|\int_{\min \{|\xi|,|\xi-\eta|\}}^{\max \{|\xi|,|\xi-\eta|\}} \frac{1}{r^{3}} d r\right| \lesssim \frac{1}{\min \{|\xi|,|\xi-\eta|\}^{2}}-\frac{1}{\max \{|\xi|,|\xi-\eta|\}^{2}} \\
& =\frac{|| \xi|-| \xi-\eta||(|\xi|+|\xi-\eta|)}{|\xi|^{2}|\xi-\eta|^{2}} \lesssim \frac{|\eta|}{\min \{|\xi|,|\xi-\eta|\}^{2} \max \{|\xi|,|\xi-\eta|\}} \\
& \lesssim \frac{|\eta|}{\langle\max \{|\xi-\eta|,|\xi|\}\rangle\langle\min \{|\xi-\eta|,|\xi|\}\rangle^{2}} .
\end{aligned}
$$

Similarly, as in (5.14),

$$
\begin{aligned}
\left|\nabla_{\eta} \Phi_{1}\right| & =\left|-p^{\prime}(\xi-\eta) \frac{\xi-\eta}{|\xi-\eta|}-p^{\prime}(\eta) \frac{\eta}{|\eta|}\right| \\
& \leq\left|p^{\prime}(\eta)-p^{\prime}(\xi-\eta)\right|+\left|p^{\prime}(\xi)\right| \frac{\eta}{|\eta|}-\frac{\xi-\eta}{|\xi-\eta|} \mid \\
& \leq\left|p^{\prime}(\eta)-p^{\prime}(\xi-\eta)\right|+2 \sqrt{2} \sin \frac{\beta}{2} \\
& \lesssim \frac{|\xi|}{\langle\max \{|\xi-\eta|,|\eta|\}\rangle\langle\min \{|\xi-\eta|,|\eta|\}\rangle^{2}}+|\sin \beta| .
\end{aligned}
$$


Using the fact that $p^{(3)}(r) \sim r^{-4}$ as $r \rightarrow+\infty$, we now compute, by (5.14), that

$$
\begin{aligned}
\left|\Delta_{\xi} \Phi_{1}\right| & =|\Delta p(\xi)-\Delta p(\xi-\eta)| \\
& =\left|p^{\prime \prime}(\xi)-p^{\prime \prime}(\xi-\eta)+2\left(\frac{p^{\prime}(\xi)}{|\xi|}-\frac{p^{\prime}(\xi-\eta)}{|\xi-\eta|}\right)\right| \\
& \lesssim \frac{|\eta|}{\langle\max \{|\xi-\eta|,|\xi|\}\rangle\langle\min \{|\xi-\eta|,|\xi|\}\rangle^{3}}+\frac{\left|p^{\prime}(\xi)-p^{\prime}(\xi-\eta)\right|}{|\xi|} \\
& +\left|\frac{1}{|\xi|}-\frac{1}{|\xi-\eta|}\right|\left|p^{\prime}(\xi-\eta)\right| \\
& \lesssim \frac{|\eta|}{\langle\max \{|\xi-\eta|,|\xi|\}\rangle\langle\min \{|\xi-\eta|,|\xi|\}\rangle^{3}} \\
& +\frac{|\eta|}{\langle\max \{|\xi-\eta|,|\xi|\}\rangle\langle\min \{|\xi-\eta|,|\xi|\}\rangle^{2}|\xi|}+\frac{|\eta|}{|\xi-\eta||\xi|} .
\end{aligned}
$$

Finally, we also get that

$$
\begin{aligned}
\Delta_{\eta} \Phi_{1} & =-\Delta_{\eta} p(\xi-\eta)-\Delta_{\eta} p(\eta) \\
& =-\left(p^{\prime \prime}(\xi-\eta)+p^{\prime \prime}(\eta)\right)-\left(\frac{2}{|\xi-\eta|} p^{\prime}(\xi-\eta)+\frac{2}{|\eta|} p^{\prime}(\eta)\right) \\
& \lesssim \frac{1}{1+|\xi-\eta|^{3}}+\frac{1}{1+|\eta|^{3}}+\frac{1}{|\eta|}+\frac{1}{|\xi-\eta|} .
\end{aligned}
$$

This ends the proof.

Proposition 5.1. Define

$$
\mathfrak{M}_{1}=\frac{|\xi||\xi-\eta \| \eta|}{\Phi_{1}\langle\xi-\eta\rangle^{2 \lambda}\langle\eta\rangle^{2 \lambda}}
$$

then if $f$ is either $\frac{\chi}{\langle\xi-\eta\rangle^{\frac{1}{2}}}$ or $\frac{\chi}{\langle\eta\rangle^{\frac{1}{2}}}$ for any cutoff function $\chi$ with support in

$$
\Omega=\{\max \{|\xi|,|\xi-\eta|,|\eta|\} \gtrsim 1\},
$$

we have that, for any $\varepsilon>0$,

$$
\begin{aligned}
& \left.\left\|\left.\mathfrak{M}_{1}\right|_{L_{\eta}^{\infty}\left(H_{\xi}^{\frac{5}{4}-\varepsilon}\right)}+\right\| \mathfrak{M}_{1}\right|_{L_{\xi}^{\infty}\left(H_{\eta}^{\frac{5}{4}-\varepsilon}\right)} \lesssim 1 \text { for } \quad \lambda>\frac{9}{8} . \\
& \left\|f \mathfrak{M}_{1}\right\|_{L_{\eta}^{\infty}\left(H_{\xi}^{\frac{3}{2}-\varepsilon}\right)}+\left\|f \mathfrak{M}_{1}\right\|_{L_{\xi}^{\infty}\left(H_{\eta}^{\frac{3}{2}-\varepsilon}\right)} \lesssim 1 \text { for } \lambda>1 .
\end{aligned}
$$

Proof of Proposition [5.1. In order to prove this proposition, we split $\mathbb{R}^{3}$ into a union of three regions: $\left\{|\xi|<\frac{1}{2}|\eta|\right\},\left\{|\eta|<\frac{1}{2}|\xi|\right\}$ and $\left\{\frac{1}{3}<\frac{|\xi|}{|\eta|}<3\right\}$. Before we start, we remark that, in the triangle defined by $\xi, \eta$ and $\xi-\eta$, we have that

$$
\frac{|\eta-\xi|}{\sin \theta}=\frac{|\xi|}{\sin \beta}=\frac{|\eta|}{\sin \gamma} \text {. }
$$

Case 1. The region $\Omega_{1}=\left\{|\xi|<\frac{1}{2}|\eta|\right\}$. In this case, $|\xi-\eta| \geq|\eta|-|\xi|>|\xi|$, so $|\xi|$ has the smallest size. We also deduce that $|\xi-\eta| \simeq|\eta|$ and consequently, since $p(\xi) \leq p(\eta)$

$$
\left|\Phi_{1}(\xi, \eta)\right|=|p(\xi)-p(\xi-\eta)-p(\eta)| \gtrsim \max \{|\eta|,|\xi-\eta|\} .
$$


We note that since $p^{\prime}$ is bounded, $\left|\nabla_{\xi, \eta} \Phi_{1}\right| \lesssim 1$ and from Lemma [5.4, we obtain that

$$
\begin{aligned}
\left|\nabla_{\xi, \eta}\left\{\frac{1}{\Phi_{1}}\right\}\right| & =\left|-\frac{\nabla_{\xi, \eta} \Phi_{1}}{\Phi_{1}^{2}}\right| \lesssim \frac{1}{\{|\eta|+|\xi-\eta|\}^{2}}, \\
\left|\Delta_{\xi}\left\{\frac{1}{\Phi_{1}}\right\}\right| & =\left|-\frac{\Delta_{\xi} \Phi_{1}}{\Phi_{1}^{2}}+2 \frac{\left|\nabla_{\xi} \Phi_{1}\right|^{2}}{\Phi_{1}^{3}}\right| \\
& \lesssim \frac{1}{\{|\eta|+|\xi-\eta|\}^{2}}\left\{\frac{1}{\left\{1+|\xi|^{3}\right\}}+\frac{1}{|\xi|}\right\}+\frac{1}{\{|\eta|+|\xi-\eta|\}^{3}} \\
& \lesssim \frac{1}{|\xi|^{3}}, \\
\left|\Delta_{\eta}\left\{\frac{1}{\Phi_{1}}\right\}\right| & =\left|-\frac{\Delta_{\eta} \Phi_{1}}{\Phi_{1}^{2}}+2 \frac{\left|\nabla_{\eta} \Phi_{1}\right|^{2}}{\Phi_{1}^{3}}\right| \\
& \lesssim \frac{1}{\{|\eta|+|\xi-\eta|\}^{2}} \frac{1}{|\eta|}+\frac{1}{\{|\eta|+|\xi-\eta|\}^{3}} \lesssim \frac{1}{|\eta|^{3}} .
\end{aligned}
$$

Recall the definition of $\chi, \varphi$ from (1.10) and denote $g=\frac{|\xi||\xi-\eta||\eta|}{\langle\xi-\eta\rangle^{2 \lambda}\langle\eta\rangle^{2 \lambda}} \varphi\left(\frac{\xi}{N}\right) \chi\left(\frac{2|\xi|}{|\eta|}\right)$, so that

$$
\begin{aligned}
\left|\mathfrak{M}_{1} \varphi\left(\frac{\xi}{N}\right) \chi\left(\frac{2|\xi|}{|\eta|}\right)\right| & \lesssim \frac{1}{\langle N\rangle^{4 \lambda-2} \varphi\left(\frac{\xi}{N}\right), \text { and }} \\
\left|\Delta_{\xi}\left\{\mathfrak{M}_{1} \varphi\left(\frac{\xi}{N}\right) \chi\left(\frac{2|\xi|}{|\eta|}\right)\right\}\right| & =\left|\Delta_{\xi}\left\{\frac{1}{\Phi_{1}}\right\} g+2 \nabla_{\xi}\left\{\frac{1}{\Phi_{1}}\right\} \cdot \nabla_{\xi} g+\frac{1}{\Phi_{1}} \Delta_{\xi} g\right| \\
& \lesssim \frac{1}{N^{2}\langle N\rangle^{4 \lambda-2}} \varphi\left(\frac{\xi}{N}\right) .
\end{aligned}
$$

We thus have that

$$
\begin{aligned}
\left\|\mathfrak{M}_{1} \varphi\left(\frac{\xi}{N}\right) \chi\left(\frac{2|\xi|}{|\eta|}\right)\right\|_{L_{\xi}^{2}} & \lesssim \frac{N^{3 / 2}}{\langle N\rangle^{4 \lambda-2}} \\
\left\|\Delta_{\xi}\left\{\mathfrak{M}_{1} \varphi\left(\frac{\xi}{N}\right) \chi\left(\frac{2|\xi|}{|\eta|}\right)\right\}\right\|_{L^{2}}+\left\|\Delta_{\eta}\left\{\mathfrak{M}_{1} \varphi\left(\frac{\eta}{N}\right) \chi\left(\frac{2|\xi|}{|\eta|}\right)\right\}\right\|_{L^{2}} & \lesssim \frac{1}{N^{1 / 2}\langle N\rangle^{4 \lambda-2}} .
\end{aligned}
$$

Interpolating between the above estimates, we get that for any $\varepsilon>0$ and any fixed fixed $\eta$,

$$
\begin{aligned}
\left\|\mathfrak{M}_{1} \chi\left(\frac{2|\xi|}{|\eta|}\right)\right\|_{\dot{H}_{\xi}^{\sigma}} & \lesssim \sum_{N}\left\|\mathfrak{M}_{1} \varphi\left(\frac{\xi}{N}\right) \chi\left(\frac{2|\xi|}{|\eta|}\right)\right\|_{\dot{H}_{\xi}^{\sigma}} \\
& \lesssim \sum_{N}\left\|\mathfrak{M}_{1} \varphi\left(\frac{\xi}{N}\right) \chi\left(\frac{2|\xi|}{|\eta|}\right)\right\|_{L^{2}}^{1-\frac{\sigma}{2}}\left\|\Delta_{\xi}\left\{\mathfrak{M}_{1} \varphi\left(\frac{\xi}{N}\right) \chi\left(\frac{2|\xi|}{|\eta|}\right)\right\}\right\|_{L^{2}}^{\frac{\sigma}{2}} \\
& \lesssim \sum_{N} \frac{N^{\frac{3}{2}-\sigma}}{\langle N\rangle^{4 \lambda-2}}
\end{aligned}
$$

which is summable in $N$ for $\lambda>1$ and $0 \leq \sigma<\frac{3}{2}$. The same proof (switching $\xi$ to $\eta$ ) works for $\sum_{N} \|\left.\mathfrak{M}_{1} \varphi\left(\frac{\eta}{N}\right) \chi\left(\frac{2|\xi|}{|\eta|}\right)\right|_{\dot{H}_{\eta}^{\sigma}}$. Both (5.17) and (5.18) are valid in this case. 
Case 2. In the region $\Omega_{2}=\left\{|\eta| \leq \frac{1}{2}|\xi|\right\}$. We note that $|\eta|$ is the smallest, and $|\xi-\eta| \simeq|\xi|$. We first claim that

$$
\left|\Phi_{1}\right| \gtrsim|\eta|\left\{\theta^{2}+\frac{|\xi|^{2}}{\langle\eta\rangle^{2}\langle\xi\rangle^{2}}\right\} \equiv|\eta|\left(\theta^{2}+d^{2}\right) .
$$

In fact, if $|\xi|$ is not the largest, then we know that $\left|\Phi_{1}\right| \geq|\eta|$ and the claim is clearly valid. If $|\xi|$ is the largest, then $\theta$ is the angle between $|\xi|$ and $|\eta|$, and $1-\cos \theta \gtrsim \theta^{2}$. Therefore we deduce (5.19) from Lemma 5.3.

We note from Lemma 5.4 that in this case,

$$
\begin{aligned}
\left|\nabla_{\xi} \Phi_{1}(\xi, \eta)\right| & \lesssim \frac{|\eta|}{1+|\xi|^{3}}+|\sin \gamma|, \\
\left|\Delta_{\xi} \Phi_{1}(\xi, \eta)\right| & \lesssim \frac{|\eta|}{1+|\xi|^{4}}+\frac{|\eta|}{|\xi|^{2}} \lesssim \frac{|\eta|}{|\xi|^{2}} .
\end{aligned}
$$

Besides, using that $\frac{\sin \gamma}{|\eta|}=\frac{\sin \beta}{|\xi|}$, the inequality above and (5.19), we can obtain that

$$
\begin{aligned}
\left|\nabla_{\xi}\left\{\frac{1}{\Phi_{1}}\right\}\right| & =\left|-\frac{\nabla_{\xi} \Phi_{1}}{\Phi_{1}^{2}}\right| \lesssim \frac{1}{|\eta|\left\{\theta^{2}+d^{2}\right\}^{2}\left(1+|\xi|^{3}\right)}+\frac{\sin \beta}{|\eta||\xi|\left\{\theta^{2}+d^{2}\right\}^{2}}, \\
\left|\Delta_{\xi}\left\{\frac{1}{\Phi_{1}}\right\}\right| & =\left|-\frac{\Delta_{\xi} \Phi_{1}}{\Phi_{1}^{2}}+2 \frac{\left|\nabla_{\xi} \Phi_{1}\right|^{2}}{\Phi_{1}^{3}}\right| \\
& \lesssim \frac{1}{|\eta|\left\{\theta^{2}+d^{2}\right\}^{2}} \frac{1}{|\xi|^{2}}+\frac{\frac{|\eta|^{2}}{\left.(1+\mid \xi)^{3}\right)^{2}}+\sin ^{2} \gamma}{|\eta|^{3}\left\{\theta^{2}+d^{2}\right\}^{3}} \\
& \lesssim \frac{1}{|\eta|\left\{\theta^{2}+d^{2}\right\}^{2}|\xi|^{2}}+\frac{1}{\left(1+|\xi|^{3}\right)^{2}|\eta|\left\{\theta^{2}+d^{2}\right\}^{3}}+\frac{\sin ^{2} \beta}{|\eta||\xi|^{2}\left\{\theta^{2}+d^{2}\right\}^{3}} .
\end{aligned}
$$

Now, for fixed $\eta$, and for any cutoff function for $\chi\left(\frac{2|\eta|}{|\xi|}\right)$, denote

$$
g=\frac{|\xi||\xi-\eta||\eta|}{\langle\xi-\eta\rangle^{2 \lambda}\langle\eta\rangle^{2 \lambda}} \varphi\left(\frac{\xi}{N}\right) \chi\left(\frac{2|\eta|}{|\xi|}\right)
$$

since $|\xi| \backsim N$ and $|\eta| \leq|\xi|$, direct computation yields

$$
\begin{aligned}
\left|\nabla_{\xi} g\right| & \lesssim \frac{1}{|\xi|} \frac{|\xi||\xi-\eta||\eta|}{\langle\xi-\eta\rangle^{2 \lambda}\langle\eta\rangle^{2 \lambda}} \mathbf{1}_{|\xi| \sim N,|\eta| \leq|\xi|}, \\
\left|\partial_{\xi}^{2} g\right| & \lesssim \frac{1}{|\xi|^{2}} \frac{|\xi||\xi-\eta||\eta|}{\langle\xi-\eta\rangle^{2 \lambda}\langle\eta\rangle^{2 \lambda}} \mathbf{1}_{|\xi| \sim N,|\eta| \leq|\xi|} .
\end{aligned}
$$

Therefore, we have that

$$
\left|\mathfrak{M}_{1} \varphi\left(\frac{\xi}{N}\right) \chi\left(\frac{2|\xi|}{|\eta|}\right)\right| \lesssim \frac{|\xi|^{2}}{\langle N\rangle^{2 \lambda}\langle\eta\rangle^{2 \lambda}\left(\theta^{2}+d^{2}\right)} \mathbf{1}_{|\xi| \backsim N,|\eta| \leq|\xi|},
$$

and by (5.20) we also have that

$$
\begin{aligned}
& \left|\Delta_{\xi}\left\{\mathfrak{M}_{1} \varphi\left(\frac{\xi}{N}\right) \chi\left(\frac{2|\xi|}{|\eta|}\right)\right\}\right| \\
& =\left|\Delta_{\xi}\left\{\frac{1}{\Phi_{1}}\right\} g+2 \nabla_{\xi}\left\{\frac{1}{\Phi_{1}}\right\} \cdot \nabla_{\xi} g+\frac{1}{\Phi_{1}} \Delta_{\xi} g\right| \\
& \lesssim \frac{|\xi|^{2} \mathbf{1}_{|\xi| \backsim N,|\eta| \leq|\xi|}}{\langle N\rangle^{2 \lambda}\langle\eta\rangle^{2 \lambda}}\left\{\frac{1}{\left\{\theta^{2}+d^{2}\right\}^{2}|\xi|^{2}}+\frac{1}{\left(1+|\xi|^{3}\right)^{2}\left\{\theta^{2}+d^{2}\right\}^{3}}+\frac{\sin ^{2} \beta}{|\xi|^{2}\left\{\theta^{2}+d^{2}\right\}^{3}}\right\} .
\end{aligned}
$$


By using $\frac{\eta}{|\eta|}$ as the north pole, we thus compute:

$$
\begin{aligned}
\int\left|\mathfrak{M}_{1} \varphi\left(\frac{\xi}{N}\right) \chi\left(\frac{2|\xi|}{|\eta|}\right)\right|^{2} d \xi & \lesssim \frac{N^{4}}{\langle N\rangle^{4 \lambda}\langle\eta\rangle^{4 \lambda}} \int_{|\xi| \sim N} \frac{|\xi|^{2} \sin \theta}{\left(\theta^{2}+d^{2}\right)^{2}} d \xi d \theta \\
& =\frac{N^{6}}{\langle N\rangle^{4 \lambda}\langle\eta\rangle^{4 \lambda}} \int_{|\xi| \sim N}\left\{\int_{\theta \leq d} \frac{\theta d \theta}{d^{4}}+\int_{\theta \geq d} \frac{d \theta}{\theta^{3}}\right\} d|\xi| \\
& \lesssim \frac{N^{7}}{\langle N\rangle^{4 \lambda}\langle\eta\rangle^{4 \lambda}} \frac{1}{d^{2}} \lesssim \frac{N^{5}}{\langle N\rangle^{4 \lambda-2}\langle\eta\rangle^{4 \lambda-2}} .
\end{aligned}
$$

Next, since $\beta=\theta+\gamma$ and $\gamma, \beta \lesssim \theta$, and $d \backsim \frac{N}{\langle\eta\rangle\langle N\rangle}$, we have

$$
\begin{aligned}
& \int\left|\Delta_{\xi}\left\{\mathfrak{M}_{1} \varphi\left(\frac{\xi}{N}\right) \chi\left(\frac{2|\xi|}{|\eta|}\right)\right\}\right|^{2} d \xi \\
& \lesssim \frac{N^{6}}{\langle N\rangle^{4 \lambda}\langle\eta\rangle^{4 \lambda}} \int_{|\xi| \sim N}\left\{\frac{1}{\left\{\theta^{2}+d^{2}\right\}^{4} N^{4}}+\frac{1}{\langle N\rangle^{12}\left\{\theta^{2}+d^{2}\right\}^{6}}+\frac{\sin ^{4} \beta}{N^{4}\left\{\theta^{2}+d^{2}\right\}^{6}}\right\} \theta d \theta d|\xi| \\
& \lesssim \frac{N^{6}}{\langle N\rangle^{4 \lambda}\langle\eta\rangle^{4 \lambda}} \times \int_{|\xi| \sim N}\left(\left\{\int_{\theta \leq d} \frac{\theta d \theta}{d^{8} N^{4}}+\int_{\theta \geq d} \frac{d \theta}{\theta^{7} N^{4}}\right\}\right. \\
& \left.+\frac{1}{\langle N\rangle^{12}}\left\{\int_{\theta \leq d} \frac{\theta d \theta}{d^{12}}+\int_{\theta \geq d} \frac{d \theta}{\theta^{11}}\right\}+\frac{1}{N^{4}}\left\{\int_{\theta \leq d} \frac{\theta^{5} d \theta}{d^{12}}+\int_{\theta \geq d} \frac{d \theta}{\theta^{7}}\right\}\right) d|\xi| \\
& \lesssim \frac{N^{3}}{\langle N\rangle^{4 \lambda}\langle\eta\rangle^{4 \lambda} d^{6}}+\frac{N^{7}}{\langle N\rangle^{4 \lambda}\langle\eta\rangle^{4 \lambda}\langle N\rangle^{12} d^{10}}+\frac{N^{3}}{\langle N\rangle^{4 \lambda}\langle\eta\rangle^{4 \lambda} d^{6}} \\
& \lesssim \frac{1}{\langle N\rangle^{4 \lambda-6}\langle\eta\rangle^{4 \lambda-6} N^{3}},
\end{aligned}
$$

where we have used the fact $\frac{1+|\eta|^{2}}{1+N^{2}} \lesssim 1$. By interpolation between (15.21) and (15.22),

$$
\begin{aligned}
\left\|\mathfrak{M}_{1} \varphi\left(\frac{\xi}{N}\right) \chi\left(\frac{2|\xi|}{|\eta|}\right)\right\|_{\dot{H}_{\xi}^{\sigma}} & \lesssim\left\|\mathfrak{M}_{1} \varphi\left(\frac{\xi}{N}\right) \chi\left(\frac{2|\xi|}{|\eta|}\right)\right\|_{L^{2}}^{1-\frac{\sigma}{2}}\left\|\Delta_{\xi}\left\{\mathfrak{M}_{1} \varphi\left(\frac{\xi}{N}\right) \chi\left(\frac{2|\xi|}{|\eta|}\right)\right\}\right\|_{L^{2}}^{\frac{\sigma}{2}} \\
& \lesssim \frac{N^{\frac{5}{2}\left(1-\frac{\sigma}{2}\right)}}{\langle N\rangle^{2 \lambda-1}\langle\eta\rangle^{2 \lambda-1}} \frac{\langle\eta\rangle^{\sigma}\langle N\rangle^{\sigma}}{N^{\frac{3}{4} \sigma}} \\
& \lesssim \frac{\langle\eta\rangle^{\sigma}\langle N\rangle^{\sigma} N^{\frac{5}{2}-2 \sigma}}{\langle N\rangle^{2 \lambda-1}\langle\eta\rangle^{2 \lambda-1}}
\end{aligned}
$$

as $|\eta| \lesssim N$. By taking $\sigma=\frac{5}{4}-\varepsilon$, this is summable for $N$ for $4 \lambda-2>\frac{5}{2}$. This concludes (5.17). On the other hand, in $\Omega$ (see (5.16)) we have $|\xi| \simeq|\xi-\eta| \geq 1$ so that $N \gtrsim 1$ and we deduce that this is summable for $N \geq 1, \sigma=3 / 2-\varepsilon$ when $\lambda>1$. This concludes (5.18).

We now turn to the $\eta$ derivatives. Using again Lemma [5.4, we have that

$$
\left|\nabla_{\eta} \Phi_{1}(\xi, \eta)\right| \lesssim \frac{|\xi|}{\langle\eta\rangle^{2}\langle\xi\rangle}+|\sin \beta|, \text { and }\left|\Delta_{\eta} \Phi_{1}(\xi, \eta)\right| \lesssim \frac{1}{|\eta|}
$$


and therefore

$$
\begin{aligned}
\left|\nabla_{\eta}\left\{\frac{1}{\Phi_{1}}\right\}\right| & =\left|-\frac{\nabla_{\eta} \Phi_{1}}{\Phi_{1}^{2}}\right| \lesssim \frac{|\xi|}{|\eta|^{2}\left\{\theta^{2}+d^{2}\right\}^{2}\langle\xi\rangle\langle\eta\rangle^{2}}+\frac{\sin \beta}{|\eta|^{2}\left\{\theta^{2}+d^{2}\right\}^{2}}, \\
\left|\Delta_{\eta}\left\{\frac{1}{\Phi_{1}}\right\}\right| & =\left|-\frac{\Delta_{\eta} \Phi_{1}}{\Phi_{1}^{2}}+2 \frac{\left|\nabla_{\eta} \Phi_{1}\right|^{2}}{\Phi_{1}^{3}}\right| \\
& \lesssim \frac{1}{|\eta|^{3}\left\{\theta^{2}+d^{2}\right\}^{2}}+\frac{1}{|\eta|^{3}\left\{\theta^{2}+d^{2}\right\}^{3}}\left\{\frac{|\xi|^{2}}{\langle\xi\rangle^{2}\langle\eta\rangle^{4}}+\sin ^{2} \beta\right\} .
\end{aligned}
$$

Define $g$ by

$$
g=\frac{|\xi||\xi-\eta||\eta|}{\langle\xi-\eta\rangle^{2 \lambda}\langle\eta\rangle^{2 \lambda}} \varphi\left(\frac{\eta}{M}\right) \chi\left(\frac{2|\eta|}{|\xi|}\right)
$$

Since $|\eta| \backsim M$ and $|\eta| \lesssim|\xi|$, direct computation yields

$$
\begin{aligned}
\left|\nabla_{\eta} g\right| & \lesssim \frac{1}{|\eta|} \frac{|\xi||\xi-\eta||\eta|}{\langle\xi-\eta\rangle^{2 \lambda}\langle\eta\rangle^{2 \lambda}} \mathbf{1}_{|\eta| \sim M,|\eta| \leq|\xi|} \\
\left|\partial_{\eta}^{2} g\right| & \lesssim \frac{1}{|\eta|^{2}} \frac{|\xi||\xi-\eta||\eta|}{\langle\xi-\eta\rangle^{2 \lambda}\langle\eta\rangle^{2 \lambda}} \mathbf{1}_{|\eta| \backsim M,|\eta| \leq|\xi|}
\end{aligned}
$$

Hence, since $\sin \beta \lesssim \sin \theta$,

$$
\begin{aligned}
\left|\Delta_{\eta}\left\{\mathfrak{M}_{1} \varphi\left(\frac{\eta}{M}\right) \chi\left(\frac{2|\xi|}{|\eta|}\right)\right\}\right|= & \left|\Delta_{\eta}\left\{\frac{1}{\Phi_{1}}\right\} g+2 \nabla_{\eta}\left\{\frac{1}{\Phi_{1}}\right\} \cdot \nabla_{\eta} g+\frac{1}{\Phi_{1}} \Delta_{\eta} g\right| \\
\lesssim & \frac{|\xi|^{2}}{M^{2}\left\{\theta^{2}+d^{2}\right\}^{2}\langle M\rangle^{2 \lambda}\langle\xi\rangle^{2 \lambda}} \\
& +\frac{|\xi|^{2}}{M^{2}\left\{\theta^{2}+d^{2}\right\}^{3}\langle M\rangle^{2 \lambda}\langle\xi\rangle^{2 \lambda}}\left\{\frac{|\xi|^{2}}{\langle\xi\rangle^{2}\langle\eta\rangle^{4}}+\sin ^{2} \theta\right\}
\end{aligned}
$$

By using $\frac{\xi}{|\xi|}$ as the north pole, and $d \backsim \frac{|\xi|}{\langle M\rangle\langle\xi\rangle}$, we thus compute that

$$
\begin{aligned}
\int_{|\eta| \sim M}\left|\mathfrak{M}_{1} \varphi\left(\frac{\eta}{M}\right) \chi\left(\frac{2|\xi|}{|\eta|}\right)\right|^{2} d \eta & \lesssim \frac{|\xi|^{4}}{\langle M\rangle^{4 \lambda}\langle\xi\rangle^{4 \lambda}} \int_{|\eta| \sim M} \frac{\sin \theta}{\left(\theta^{2}+d^{2}\right)^{2}} d \eta d \theta \\
& \lesssim \frac{|\xi|^{4} M^{2}}{\langle M\rangle^{4 \lambda}\langle\xi\rangle^{4 \lambda}} \int_{|\eta| \sim M}\left\{\int_{\theta \leq d} \frac{\theta d \theta}{d^{4}}+\int_{\theta \geq d} \frac{d \theta}{\theta^{3}}\right\} d|\eta| \\
& \lesssim \frac{|\xi|^{4} M^{3}}{\langle M\rangle^{4 \lambda}\langle\xi\rangle^{4 \lambda}} \frac{1}{d^{2}} \lesssim \frac{|\xi|^{2} M^{3}}{\langle M\rangle^{4 \lambda-2}\langle\xi\rangle^{4 \lambda-2}}
\end{aligned}
$$


Next, since $\beta=\theta+\gamma$ and $\gamma, \beta \lesssim \theta$, and we have that

$$
\begin{aligned}
& \int_{|\eta| \sim M}\left|\Delta_{\eta}\left\{\mathfrak{M}_{1} \varphi\left(\frac{\eta}{M}\right) \chi\left(\frac{2|\xi|}{|\eta|}\right)\right\}\right|^{2} d \eta \\
& \lesssim \frac{1}{M^{2}\langle M\rangle^{4 \lambda}\langle\xi\rangle^{4 \lambda}} \\
& \times \int_{|\eta| \backsim M}\left\{\frac{|\xi|^{4}}{\left\{\theta^{2}+d^{2}\right\}^{4}}+\frac{|\xi|^{8}}{\left\{\theta^{2}+d^{2}\right\}^{6}\langle\xi\rangle^{4}\langle\eta\rangle^{8}}+\frac{|\xi|^{4} \sin ^{4} \theta}{\left\{\theta^{2}+d^{2}\right\}^{6}}\right\} \theta d \theta d|\eta| \\
& \lesssim \frac{|\xi|^{4}}{M^{2}\langle M\rangle^{4 \lambda}\langle\xi\rangle^{4 \lambda}} \int_{|\eta| \backsim M}\left(\left\{\int_{\theta \lesssim d} \frac{\theta d \theta}{d^{8}}+\int_{\theta \geq d} \frac{d \theta}{\theta^{7}}\right\}\right. \\
& \left.+\frac{|\xi|^{8}}{\langle\xi\rangle^{4}\langle M\rangle^{8}}\left\{\int_{\theta \leq d} \frac{\theta d \theta}{d^{12}}+\int_{\theta \geq d} \frac{d \theta}{\theta^{11}}\right\}+\left\{\int_{\theta \lesssim d} \frac{\theta^{5} d \theta}{d^{12}}+\int_{\theta \geq d} \frac{d \theta}{\theta^{7}}\right\}\right) d|\eta| \\
& \lesssim \frac{|\xi|^{4}}{M\langle M\rangle^{4 \lambda}\langle\xi\rangle^{4 \lambda} d^{6}}+\frac{|\xi|^{4}}{M\langle M\rangle^{4 \lambda+8}\langle\xi\rangle^{4 \lambda+4} d^{10}}+\frac{1}{M\langle M\rangle^{4 \lambda}\langle\xi\rangle^{4 \lambda} d^{6}} \\
& \lesssim \frac{1}{M\langle M\rangle^{4 \lambda-6}\langle\xi\rangle^{4 \lambda-6}|\xi|^{2}} .
\end{aligned}
$$

Interpolating between (5.23) and (5.24), we obtain

$$
\begin{aligned}
\left\|\mathfrak{M}_{1} \varphi\left(\frac{\eta}{M}\right) \chi\left(\frac{2|\xi|}{|\eta|}\right)\right\|_{\dot{H}_{\eta}^{\sigma}} & \lesssim\left\|\mathfrak{M}_{1} \varphi\left(\frac{\eta}{M}\right) \chi\left(\frac{2|\xi|}{|\eta|}\right)\right\|_{L^{2}}^{1-\frac{\sigma}{2}}\left\|\Delta_{\eta}\left\{\mathfrak{M}_{1} \varphi\left(\frac{\eta}{M}\right) \chi\left(\frac{2|\xi|}{|\eta|}\right)\right\}\right\|_{L^{2}}^{\frac{\sigma}{2}} \\
& \lesssim\left\{\frac{|\xi|^{2} M^{3}}{\langle M\rangle^{4 \lambda-2}\langle\xi\rangle^{4 \lambda-2}}\right\}^{\frac{1}{2}-\frac{\sigma}{4}}\left\{\frac{1}{M\langle M\rangle^{4 \lambda-6}\langle\xi\rangle^{4 \lambda-6}|\xi|^{2}}\right\}^{\frac{\sigma}{4}} \\
& \lesssim \frac{M^{\frac{3}{2}-\sigma}}{\langle M\rangle^{2 \lambda-1-\sigma}\langle\xi\rangle^{2 \lambda-1-\sigma}|\xi|^{\sigma-1}}
\end{aligned}
$$

By taking $\sigma=\frac{5}{4}-\varepsilon$, this is summable in $M$ if $2 \lambda-1-\sigma>0$ and we conclude (5.17). On the other hand, in $\Omega$, we have $|\xi| \geq 1$ so that by taking $\sigma=\frac{3}{2}-\varepsilon$, this is summable for $M \geq 1$ if $\lambda>1$ and if $f=\frac{1}{\langle\xi-\eta\rangle^{\frac{1}{2}}}$ or $\frac{1}{\langle\eta\rangle^{\frac{1}{2}}}$.

Case 3 Region $\Omega_{3}=\left\{\frac{1}{3}<\frac{|\xi|}{|\eta|}<3\right\}$.

In this region, we have $|\xi-\eta| \leq 4 \min (|\xi|,|\eta|),|\xi| \simeq|\eta|$ are of the order of the longest side and $\sin \gamma \simeq \sin \beta$. Therefore

$$
\left|\Phi_{1}\right| \geq|\xi-\eta|\left(\gamma^{2}+\beta^{2}+\frac{|\xi|^{2}+|\eta|^{2}}{\left(1+|\xi|^{2}+|\eta|^{2}\right)\langle\xi-\eta\rangle^{2}}\right) \equiv|\xi-\eta|\left(\gamma^{2}+\beta^{2}+d_{1}^{2}\right) .
$$

The above lower bound is trivial if $|\xi|$ is not the largest. If $|\xi|$ is the largest and $|\xi-\eta|$ is not the smallest, then $\xi, \eta, \xi-\eta$ are all comparable so that $\gamma \simeq \theta \simeq \pi-\beta$ and from (5.7),

$$
\left|\Phi_{1}\right| \gtrsim \theta^{2}|\eta|+\frac{|\eta|}{1+|\eta|^{2}} \gtrsim|\xi-\eta|\left(\gamma^{2}+\beta^{2}+d_{1}^{2}\right) .
$$

Finally, when $|\xi-\eta|$ is the smallest, this follows from (5.7). Moreover, from (5.10) and (5.11),

$$
\left|\partial_{\xi, \eta} \Phi_{1}(\xi, \eta)\right| \lesssim \frac{|\eta|}{\langle\eta\rangle\langle\xi-\eta\rangle^{2}}+|\sin \gamma|, \quad\left|\Delta_{\xi, \eta} \Phi_{1}(\xi, \eta)\right| \lesssim \frac{1}{|\xi-\eta|}
$$


and therefore,

$$
\begin{aligned}
\left|\nabla_{\xi, \eta}\left\{\frac{1}{\Phi_{1}}\right\}\right|= & \left|-\frac{\nabla_{\xi} \Phi_{1}}{\Phi_{1}^{2}}\right| \\
\lesssim & \frac{|\eta|}{|\xi-\eta|^{2}\left(\beta^{2}+\gamma^{2}+d_{1}^{2}\right)^{2}\langle\eta\rangle\langle\xi-\eta\rangle^{2}}+\frac{\sin \gamma}{|\xi-\eta|^{2}\left(\beta^{2}+\gamma^{2}+d_{1}^{2}\right)^{2}} \\
\left|\Delta_{\xi, \eta}\left\{\frac{1}{\Phi_{1}}\right\}\right|= & \left|-\frac{\Delta_{\xi} \Phi_{1}}{\Phi_{1}^{2}}+2 \frac{\left|\nabla_{\xi} \Phi_{1}\right|^{2}}{\Phi_{1}^{3}}\right| \\
\lesssim & \frac{1}{|\xi-\eta|^{3}\left(\beta^{2}+\gamma^{2}+d_{1}^{2}\right)^{2}}+\frac{|\eta|^{2}+|\xi|^{2}}{|\xi-\eta|^{3}\left(\beta^{2}+\gamma^{2}+d_{1}^{2}\right)^{3}\langle\eta\rangle^{2}\langle\xi-\eta\rangle^{4}} \\
& +\frac{\sin ^{2} \gamma+\sin ^{2} \beta}{|\xi-\eta|^{3}\left(\beta^{2}+\gamma^{2}+d_{1}^{2}\right)^{3}} .
\end{aligned}
$$

For fixed $\eta$ and a dyadic number $N$, denote

$$
g=\frac{|\xi||\xi-\eta||\eta|}{\langle\xi-\eta\rangle^{2 \lambda}\langle\eta\rangle^{2 \lambda}} \varphi\left(\frac{\xi-\eta}{N}\right) \varphi\left(\sqrt{\frac{|\eta|}{|\xi|}}\right)
$$

As before, direct computation yields

$$
\begin{aligned}
\left|\nabla_{\xi, \eta} g\right| & \lesssim \frac{1}{|\xi-\eta|} \frac{|\xi||\xi-\eta||\eta|}{\langle\xi-\eta\rangle^{2 \lambda}\langle\eta\rangle^{2 \lambda}} \mathbf{1}_{|\xi-\eta| \sim N,|\xi| \sim|\eta|} \\
\left|\partial_{\xi, \eta}^{2} g\right| & \lesssim \frac{1}{|\xi-\eta|^{2}} \frac{|\xi||\xi-\eta||\eta|}{\langle\xi-\eta\rangle^{2 \lambda}\langle\eta\rangle^{2 \lambda}} \mathbf{1}_{|\xi-\eta| \sim N,|\xi| \sim|\eta|} .
\end{aligned}
$$

Therefore,

$$
\left|\mathfrak{M}_{1} \varphi\left(\frac{\xi-\eta}{N}\right) \varphi\left(\sqrt{\frac{|\eta|}{|\xi|}}\right)\right| \lesssim \frac{|\eta|^{2} \mathbf{1}_{|\xi-\eta| \sim N,|\xi| \sim|\eta|}}{\langle N\rangle^{2 \lambda}\langle\eta\rangle^{2 \lambda}\left(\beta^{2}+d_{1}^{2}\right)}
$$

and

$$
\begin{aligned}
& \left|\Delta_{\xi}\left\{\mathfrak{M}_{1} \varphi\left(\frac{\xi-\eta}{N}\right) \varphi\left(\sqrt{\frac{|\eta|}{|\xi|}}\right)\right\}\right| \\
\lesssim & \left\{\frac{|\eta|^{2}}{\left(\beta^{2}+d_{1}^{2}\right)^{2}}+\frac{|\eta|^{4}}{\left(\beta^{2}+d_{1}^{2}\right)^{3}\langle\eta\rangle^{2}\langle\xi-\eta\rangle^{4}}+\frac{|\eta|^{2} \sin ^{2} \beta}{\left(\beta^{2}+d_{1}^{2}\right)^{3}}\right\} \frac{\mathbf{1}_{|\xi-\eta| \backsim N,|\xi| \backsim|\eta|}}{N^{2}\langle N\rangle^{2 \lambda}\langle\eta\rangle^{2 \lambda}} .
\end{aligned}
$$

By using $-\frac{\eta}{|\eta|}$ as the north pole, and $d_{1} \backsim \frac{|\eta|}{\langle\eta\rangle\langle N\rangle}$, we thus compute from (5.25):

$$
\begin{aligned}
& \int\left|\mathfrak{M}_{1} \varphi\left(\frac{\xi-\eta}{N}\right) \varphi\left(\sqrt{\frac{|\eta|}{|\xi|}}\right)\right|^{2} d \xi \\
& \lesssim \frac{|\eta|^{4} N^{2}}{\langle N\rangle^{4 \lambda}\langle\eta\rangle^{4 \lambda}} \int_{|\xi-\eta| \sim N} \frac{\sin \beta}{\left(\beta^{2}+d_{1}^{2}\right)^{2}} d|\xi| d \beta \\
& =\frac{|\eta|^{4} N^{3}}{\langle N\rangle^{4 \lambda}\langle\eta\rangle^{4 \lambda}} \int_{|\xi-\eta| \sim N}\left\{\int_{\beta \leq d_{1}} \frac{\beta d \beta}{d_{1}^{4}}+\int_{\beta \geq d} \frac{d \beta}{\beta^{3}}\right\} d|\xi| \\
& \lesssim \frac{|\eta|^{4} N^{3}}{\langle N\rangle^{4 \lambda}\langle\eta\rangle^{4 \lambda}} \frac{1}{d_{1}^{2}} \lesssim \frac{|\eta|^{2} N^{3}}{\langle N\rangle^{4 \lambda-2}\langle\eta\rangle^{4 \lambda-2}} .
\end{aligned}
$$


Next, we have that

$$
\begin{aligned}
& \int_{|\xi-\eta| \sim N}\left|\Delta_{\xi}\left\{\mathfrak{M}_{1} \varphi\left(\frac{\xi-\eta}{N}\right) \varphi\left(\sqrt{\frac{|\eta|}{|\xi|}}\right)\right\}\right|^{2} d \xi \\
& \lesssim \frac{|\eta|^{4}}{\langle N\rangle^{4 \lambda}\langle\eta\rangle^{4 \lambda} N^{4}} \int_{|\xi-\eta| \sim N}\left\{\frac{1}{\left(\beta^{2}+d_{1}^{2}\right)^{4}}+\frac{|\eta|^{4}}{\left(\beta^{2}+d_{1}^{2}\right)^{6}\langle\eta\rangle^{4}\langle N\rangle^{8}}+\frac{\beta^{4}}{\left(\beta^{2}+d_{1}^{2}\right)^{6}}\right\} d(\xi-\eta) \\
& \lesssim \frac{|\eta|^{4} N^{2}}{\langle N\rangle^{4 \lambda}\langle\eta\rangle^{4 \lambda} N^{4}} \int_{|\xi-\eta| \sim N} d|\xi-\eta|\left(\left\{\int_{\beta \leq d_{1}} \frac{\beta d \beta}{d_{1}^{8}}+\int_{\beta \geq d_{1}} \frac{d \beta}{\beta^{7}}\right\}\right. \\
& \left.+\frac{|\eta|^{4}}{\langle\eta\rangle^{4}\langle N\rangle^{8}}\left\{\int_{\beta \leq d_{1}} \frac{\beta d \beta}{d_{1}^{12}}+\int_{\beta \geq d_{1}} \frac{d \beta}{\beta^{11}}\right\}+\left\{\int_{\beta \leq d_{1}} \frac{\beta^{5} d \beta}{d_{1}^{12}}+\int_{\beta \geq d_{1}} \frac{d \beta}{\beta^{7}}\right\}\right) \\
& \lesssim \frac{|\eta|^{4}}{\langle N\rangle^{4 \lambda}\langle\eta\rangle^{4 \lambda} N d^{6}}+\frac{|\eta|^{4}}{\langle N\rangle^{4 \lambda+8}\langle\eta\rangle^{4 \lambda+4} N d_{1}^{10}}+\frac{1}{\langle N\rangle^{4 \lambda} N\langle\eta\rangle^{4 \lambda} d_{1}^{6}} \\
& \lesssim \frac{1}{\langle N\rangle^{4 \lambda-6}\langle\eta\rangle^{4 \lambda-6}|\eta|^{2} N} .
\end{aligned}
$$

Interpolating between (5.27) and (5.28), we have

$$
\begin{aligned}
& \left\|\mathfrak{M}_{1} \varphi\left(\frac{\xi-\eta}{N}\right) \varphi\left(\sqrt{\frac{|\eta|}{|\xi|}}\right)\right\|_{\dot{H}_{\xi}^{\sigma}} \\
\lesssim & \left\|\mathfrak{M}_{1} \varphi\left(\frac{\xi-\eta}{N}\right) \varphi\left(\sqrt{\frac{|\eta|}{|\xi|}}\right)\right\|_{L^{2}}^{1-\frac{\sigma}{2}}\left\|\Delta_{\xi}\left\{\mathfrak{M}_{1} \varphi\left(\frac{\xi-\eta}{N}\right) \varphi\left(\sqrt{\frac{|\eta|}{|\xi|}}\right)\right\}\right\|_{L^{2}}^{\frac{\sigma}{2}} \\
\lesssim & \left\{\frac{|\eta|^{2} N^{3}}{\langle N\rangle^{4 \lambda-2}\langle\eta\rangle^{4 \lambda-2}}\right\}^{\frac{1}{2}-\frac{\sigma}{4}}\left\{\frac{1}{\langle N\rangle^{4 \lambda-6}\langle\eta\rangle^{4 \lambda-6}|\eta|^{2} N}\right\}^{\frac{\sigma}{4}} \\
\lesssim & \frac{N^{\frac{3}{2}-\sigma}}{\langle N\rangle^{2 \lambda-1-\sigma}\langle\eta\rangle^{2 \lambda-1-\sigma}|\eta|^{\sigma-1}}
\end{aligned}
$$

as $N \lesssim|\eta|$. By taking $\sigma=\frac{5}{4}-\varepsilon$, this is summable for $N$ when $4 \lambda-2>\frac{5}{2}$ hence we deduce (5.17). On the other hand, in $\Omega$, we know that $|\eta| \simeq|\xi| \geq 1$. Hence for $f=\frac{1}{\langle\xi-\eta\rangle^{\frac{1}{2}}}$ or $\frac{1}{\langle\eta\rangle^{\frac{1}{2}}}$, we can take $\sigma=\frac{3}{2}-\varepsilon$ and still get a convergent series. (5.18) therefore follows. The $\eta$ derivatives can be controlled similarly since we had the same control.

\section{The $L^{10}$ Bound AND END OF THE PROOF}

6.1. Estimating the $L^{10}$ bound. Using the results of Section 5 , we can now estimate the last part of the $X$ norm.

Proposition 6.1. Let $\alpha$ be a solution of (3.14), then

$$
\sup _{t \geq 0}(1+t)^{\frac{16}{15}}\|\alpha(t)\|_{W^{k, 10}} \lesssim\left\|\alpha_{0}\right\|_{Y}+\|\alpha\|_{X}^{2} .
$$

Proof. We use Theorem 5.1 and Proposition 5.1 to control the nonlinear terms appearing in (3.14). Our strategy is first to establish the Proposition for $\Phi_{1}$, and then we use symmetry to conclude all the other cases. We first deal with the cubic terms as follows. We let

$$
A(\xi-\eta)=\frac{n_{2}(\xi-\eta)}{|\xi-\eta|}\langle\xi-\eta\rangle^{2 \lambda} \text { and } B(\eta)=\frac{n_{3}(\eta)}{|\eta|}\langle\eta\rangle^{2 \lambda}
$$


We first apply Proposition 2.1 to get that, for a typical term,

$$
\begin{aligned}
& \left\|(1-\Delta)^{\frac{k}{2}} \mathcal{F}^{-1}\left\{\int_{0}^{t} e^{i(t-s) p(|\xi|)} \frac{i m^{1}(\xi, \eta)}{\Phi_{1}} \hat{\alpha}(s, \xi-\eta) \hat{h}(\alpha)(s, \eta) d s\right\}\right\|_{L^{10}} \\
& \lesssim \int_{0}^{t} \frac{1}{(1+t-s)^{\frac{16}{15}}}\left\|\mathcal{F}_{\xi}^{-1}\left\{n_{1}(\xi)\langle\xi\rangle^{k} \int_{\mathbb{R}^{3}} \frac{|\xi|}{\Phi_{1}} n_{2}(\xi-\eta) \hat{\alpha}(\xi-\eta) n_{3}(\eta) \hat{h}(\eta) d \eta\right\}\right\|_{W^{\frac{12}{5}, \frac{10}{9}}} d s \\
& \lesssim \int_{0}^{t} \frac{1}{(1+t-s)^{\frac{16}{15}}}\left\|\mathcal{F}_{\xi}^{-1}\left\{\langle\xi\rangle^{k+\frac{12}{5}} \int_{\mathbb{R}^{3}} \mathfrak{M}_{1} A(\xi-\eta) \hat{\alpha}(\xi-\eta) B(\eta) \hat{h}(\eta)\right\}\right\|_{L^{\frac{10}{9}}} d s \\
& \lesssim \int_{0}^{t} \frac{1}{(1+t-s)^{\frac{16}{15}}}\left(\|A(|\nabla|) \alpha\|_{H^{k+\frac{12}{5}}}\|B(|\nabla|) \beta\|_{L^{l_{2}}}+\|A(|\nabla|) \alpha\|_{L^{l_{2}}}\|B(|\nabla|) \beta\|_{H^{k+\frac{12}{5}}}\right) d s \\
& \lesssim \int_{0}^{t} \frac{1}{(1+t-s)^{\frac{16}{15}}}\left(\|\alpha\|_{H^{-1} \cap H^{k+2 \lambda+\frac{7}{5}}}\left\||\nabla|^{-1} \beta\right\|_{H^{3}}+\|\alpha\|_{H^{-1} \cap H^{2}}\left\||\nabla|^{-1} \beta\right\|_{H^{k+2 \lambda+\frac{12}{5}}}\right) d s \\
& \lesssim(1+t)^{-\frac{16}{15}}\|\alpha\|_{X}^{3}
\end{aligned}
$$

since $k \geq 2 \lambda+\frac{7}{5}$. Here we have applied Lemma 5.1 around $s=\frac{5}{4}-\varepsilon$, Proposition 5.1 and Theorem 5.1 with $l_{1}=10, b=\infty$, and $l_{2}=\frac{60}{29+20 \varepsilon}>2$. To finish the analysis of the cubic term, we also need to control the cubic term pre-normal form in (3.14). We use the fact that $e^{i t p(|\nabla|)}$ is a unitary operator on $H^{k}$ and (3.17) to get that

$$
\begin{aligned}
\left\|\mathcal{F}^{-1} \int_{0}^{t} e^{i(t-s) p(\xi)} \hat{\mathcal{N}}_{1}(\alpha)(s) d s\right\|_{W^{k, 10}} & \lesssim \int_{0}^{t}\left\|e^{i(t-s) p(|\nabla|)} \mathcal{N}_{1}(\alpha)(s)\right\|_{H^{k+2}} d s \\
& \lesssim \int_{0}^{t}\left\||\nabla|^{-1} \mathcal{N}_{1}(\alpha)(s)\right\|_{H^{k+3}} d s \\
& \lesssim\|\alpha\|_{X}^{2}
\end{aligned}
$$

To estimate the integrated term $\mathfrak{B}$ in (3.14), we need to separate the regions. First we control the integrated part when all the terms are small,

$$
M=\max (|\xi|,|\xi-\eta|,|\eta|)<3 .
$$

To do this, we first note that Sobolev's embedding $\dot{H}^{\frac{6}{5}} \subset L^{10}$, and the fact that for bounded $\xi,|\xi|^{\frac{6}{5}} \lesssim|\xi| \lesssim|\xi-\eta|+|\eta|$ to get?

$$
\begin{aligned}
& \|\mathfrak{B}(\alpha(t), \alpha(t))\|_{L^{10}} \\
& \lesssim\left\|\mathcal{F}_{\xi}^{-1}\left\{|\xi|^{\frac{6}{5}} \int_{\mathbb{R}^{3}} \frac{|\xi||\xi-\eta \|| \eta \mid}{i \Phi_{1}} \frac{m}{|\xi|} \frac{\hat{\alpha}(t, \xi-\eta)}{|\xi-\eta|} \frac{\hat{\alpha}(t, \eta)}{|\eta|} d \eta\right\}\right\|_{L^{2}} \\
& \lesssim\left\|\int_{\mathbb{R}^{3}} \mathfrak{M}_{1} n_{1}(\xi) n_{2}(\xi-\eta) n_{3}(\eta)\langle\xi-\eta\rangle^{2 \lambda} \hat{\alpha}(t, \xi-\eta) \frac{\langle\eta\rangle^{2 \lambda} \hat{\alpha}(t, \eta)}{|\eta|} d \eta\right\|_{L^{2}} \\
& \lesssim\left\|n_{2}(|\nabla|) \alpha\right\|_{L^{10}}\left\||\nabla|^{-1} n_{3}(|\nabla|) \alpha\right\|_{L^{2}} \lesssim\|\alpha\|_{X}\|\alpha\|_{L^{10}}
\end{aligned}
$$

where we have applied Proposition 5.1 for $\mathfrak{M}_{1}$ for $s=\frac{5}{4}-\varepsilon$, Lemma 5.1, and Lemma 5.2 with $l_{1}=2, b=\infty, l_{2}=10$ and $l_{3}=\frac{60}{29+20 \varepsilon}>2$.

Next we deal with the case when one of the frequencies is large $M>1$. This can happen in two cases. First, if $|\xi| \leq 1, M>1$. In this case, we have $|\eta| \simeq|\xi-\eta| \geq 1$.

\footnotetext{
${ }^{2}$ Here we forget the difference between $n_{2}$ and $n_{3}$ and treat the terms as symmetric.
} 
We bound the $L^{10}$ norm by the $L^{2}$ norm via Sobolev's inequality (with bounded $\xi$ ) to get

$$
\begin{aligned}
\|\mathfrak{B}\|_{L^{10}} & \lesssim\left\|\mathcal{F}_{\xi}^{-1} \int_{\mathbb{R}^{3}} \frac{m(\xi, \eta)}{i \Phi_{1}} \chi \hat{\alpha}(t, \xi-\eta) \hat{\alpha}(t, \eta) d \eta\right\|_{L^{10}} \\
& \lesssim\left\|\mathcal{F}_{\xi}^{-1} \int_{\mathbb{R}^{3}} n_{1}(\xi) f \mathfrak{M}_{j} n_{2}(\xi-\eta) \frac{\langle\xi-\eta\rangle^{2 \lambda+\frac{1}{4}} \hat{\alpha}(t, \xi-\eta)}{|\xi-\eta|} n_{3}(\eta) \frac{\langle\eta\rangle^{2 \lambda+\frac{1}{4}} \hat{\alpha}(t, \eta)}{|\eta|} d \eta\right\|_{L^{10}} \\
& \lesssim\left\|\mathcal{F}_{\xi}^{-1} \int_{\mathbb{R}^{3}} f \mathfrak{M}_{j} n_{2}(\xi-\eta) \frac{\langle\xi-\eta\rangle^{2 \lambda+\frac{1}{4}} \hat{\alpha}(t, \xi-\eta)}{|\xi-\eta|} n_{3}(\eta) \frac{\langle\eta\rangle^{2 \lambda+\frac{1}{4}} \hat{\alpha}(t, \eta)}{|\eta|} d \eta\right\|_{L^{2}} \\
& \lesssim\|\alpha\|_{H^{2}}\left\|n_{3}(|\nabla|) \alpha\right\|_{W^{2, \frac{3}{\varepsilon}}} \lesssim\|\alpha\|_{X}\|\alpha\|_{W^{3,10}} .
\end{aligned}
$$

We have applied Proposition 5.1 with $s=\frac{3}{2}-\varepsilon$, Lemma 5.1 around $s=\frac{3}{2}-\varepsilon$ and Lemma 5.2 with $s=\frac{3}{2}-\varepsilon, b=\infty, l_{1}=2, l_{2}=10$ and $l_{3}=\frac{15}{6+5 \varepsilon}>2$. This concludes the estimates in the region $\{|\xi| \leq 1\} \cap\{M \geq 1\}$.

The other case is included in the region

$$
\Omega=\{|\eta| \leq 2|\xi-\eta|,|\xi| \geq 1 / 2\} \cup\{|\eta|>|\xi-\eta|,|\xi| \geq 1 / 2\}
$$

and leads to the worst loss in derivatives (whereas the region when all frequencies are small leads to the loss of smoothness of the multiplier and and hence to the loss of decay in time). In the case $|\eta| \leq 2|\xi-\eta|$, we choose $f=\frac{\chi}{\langle\eta\rangle^{\frac{1}{2}}}$ in Proposition [5.1] We apply Lemma 5.1 to deduce that

$$
\frac{|\xi|^{k+\frac{6}{5}}}{\langle\xi-\eta\rangle^{k+\frac{6}{5}}} \mathfrak{M}_{1} f \in M_{\xi, \eta}^{\frac{3}{2}-\varepsilon} .
$$

Hence

$$
\begin{aligned}
& \left\||\nabla|^{k} \mathfrak{B}(\alpha, \alpha)\right\|_{L^{10}} \\
& \lesssim\left\|\mathcal{F}_{\xi}^{-1}\left\{|\xi|^{k} n_{1}(\xi) \int_{\mathbb{R}^{3}} f \mathfrak{M}_{j} n_{2}(\xi-\eta) \frac{\langle\xi-\eta\rangle^{2 \lambda} \hat{\alpha}(\xi-\eta)}{|\xi-\eta|} n_{3}(\eta) \frac{\langle\eta\rangle^{2 \lambda+\frac{1}{2}} \hat{\alpha}(\eta)}{|\eta|} d \eta\right\}\right\|_{L^{10}} \\
& \lesssim\left\|\mathcal{F}^{-1} \int_{\mathbb{R}^{3}}\left[\frac{|\xi|^{k+\frac{6}{5}}}{\langle\xi-\eta\rangle^{k+\frac{6}{5}}} \mathfrak{M}_{j} f\right] n_{2}(\xi-\eta) \frac{\langle\xi-\eta\rangle^{k+2 \lambda+\frac{6}{5}} \hat{\alpha}(\xi-\eta)}{|\xi-\eta|} n_{3}(\eta) \frac{\langle\eta\rangle^{2 \lambda+\frac{1}{2}}}{|\eta|} \hat{\alpha}(\eta) d \eta\right\|_{L^{2}} \\
& \lesssim\left\|\frac{|\xi|^{k+\frac{6}{5}}}{\langle\xi-\eta\rangle^{k+\frac{6}{5}}} \mathfrak{M}_{j} f\right\|_{\mathcal{M}_{\xi, \eta}^{\frac{3}{2}-\varepsilon}}\left\||\nabla|^{k+\frac{11}{5}+2 \delta} n_{2}(|\nabla|) \alpha\right\|_{L^{l_{3}}}\left\|\frac{(1-\Delta)^{2}}{|\nabla|} n_{3}(|\nabla|) \alpha\right\|_{L^{l_{2}}} \\
& \lesssim\left\||\nabla|^{k+\frac{11}{5}+2 \delta} \alpha\right\|_{L^{l_{2}}}\left\|\frac{(1-\Delta)^{2}}{|\nabla|} \alpha\right\|_{L^{l_{3}}} \text {. }
\end{aligned}
$$

We have applied Lemma with $s=\frac{3}{2}-\varepsilon, \frac{1}{l_{2}}=\frac{14}{50}+\frac{17}{15} \varepsilon$ and $\frac{1}{l_{3}}=\frac{11}{50}-\frac{4}{5} \varepsilon$. Now, using Bernstein estimates, we compute that

$$
\begin{aligned}
\left\|P_{\leq 1} \frac{(1-\Delta)^{2}}{|\nabla|} \alpha\right\|_{L^{l_{2}}} & \lesssim \sum_{N \leq 1} N^{-1}\left\|P_{N} \alpha\right\|_{L^{l_{2}}} \\
& \lesssim \sum_{N \leq 1} N^{-1} N^{3\left(\frac{1}{l_{4}}-\frac{1}{l_{2}}\right)}\left\|P_{N} \alpha\right\|_{L^{l_{4}}} \\
& \lesssim \sum_{N \leq 1} N^{\varepsilon}\left(N^{-1}\left\|P_{N} \alpha\right\|_{L^{2}}\right)^{1-\sigma}\left\|P_{N} \alpha\right\|_{L^{10}}^{\sigma} \lesssim\|\alpha\|_{X}^{1-\sigma}\|\alpha\|_{L^{10}}^{\sigma}
\end{aligned}
$$


for

$$
\sigma=\frac{5}{11}\left(\frac{3}{b}-2 \varepsilon\right)=\frac{3}{10}+\frac{2 \varepsilon}{11}, \text { and } \frac{1}{l_{4}}=\frac{1}{2}-\frac{2 \sigma}{5}=\frac{19}{50}-\frac{4}{55} \varepsilon
$$

while for the high frequencies, we have that

$$
\begin{aligned}
\left\|P_{\geq 1} \frac{(1-\Delta)^{2}}{|\nabla|} \alpha\right\|_{L^{l_{2}}} & \lesssim\left\|(1-\Delta)^{\frac{3}{2}} \alpha\right\|_{L^{10}}^{\sigma}\left\|(1-\Delta)^{\frac{3}{2}} \alpha\right\|_{L^{l_{5}}}^{1-\sigma} \\
& \lesssim\|\alpha\|_{W^{3,10}}^{\sigma}\|\alpha\|_{X}^{1-\sigma}
\end{aligned}
$$

for $\frac{1}{l_{5}}=(1 / 4+184 \varepsilon) /(7 / 10-2 / 11 \varepsilon)$. Independently, we have that

$$
\begin{aligned}
\left\||\nabla|^{k+\frac{11}{5}+2 \delta} \alpha\right\|_{L^{l_{3}}} & \lesssim\left\|(1-\Delta)^{\frac{k}{2}} \alpha\right\|_{L^{10}}^{1-\sigma}\left\|(1-\Delta)^{\frac{k}{2}+\left(\frac{11}{10}+2 \delta\right) \frac{1}{\sigma}} \alpha\right\|_{L^{2}}^{\sigma} \\
& \lesssim\|\alpha\|_{W^{k, 10}}^{1-\sigma}\|\alpha\|_{X}^{\sigma}
\end{aligned}
$$

provided that $k>11 /(5 \sigma)=\frac{22}{3}+\varepsilon$.

In the case $|\eta|>|\xi-\eta|$ we proceed similarly with $f=\frac{\chi}{\langle\xi-\eta\rangle^{1 / 2}}$. We therefore conclude the Proposition for $\Phi_{1}$.

We now have completed the proof for $j=1$ by Lemma and Theorem. To establish (6.1) for $j \neq 1$, we note that the proposition is clearly valid for $\Phi_{2}$ because the proof in Case 1 shows that Proposition 5.1 is also valid in this easier case (indeed, $\left.\left|\Phi_{2}\right| \gtrsim \max (|\xi|,|\xi-\eta|,|\eta|)\right)$. For $\Phi_{4}$, we note $\Phi_{4}(\xi, \eta)=-\Phi_{1}(\eta, \xi)$, and repeat the same proof in light of Proposition 5.1. Finally, for $\Phi_{3}(\xi, \eta)=-\Phi_{1}(\xi-\eta, \xi)$, we make a change of integration variable $\eta \rightarrow \xi-\eta$ in the integrations in both the cubic terms and $\mathfrak{B}$ and get back to the previous case. We thus conclude the proof.

6.2. End of the proof. Now, we are ready to finish the proof of Theorem 1.1

Proof of Theorem 1.1. The existence of a local regular solution $\left.\beta \in C\left(0, T^{*}\right), X\right)$ follows from the standard method of Kato [15]. Combining Proposition 4.1 and Proposition 6.1 we obtain that

$$
\|\beta\|_{X} \lesssim\|\alpha(0)\|_{Y}+\|\beta\|_{X}^{2}
$$

so that if $\|\alpha(0)\|_{Y}$ is sufficiently small, we get a global bound on the $X$-norm of the solution, which implies that $T^{*}=\infty$ and gives a global bound on the $X$-norm of $\rho$ and $v$. This ends the proof.

\section{REFERENCES}

[1] Chen, Gui-Qiang; Jerome, J. W. and Wang, D., Compressible Euler-Maxwell equations. Proceedings of the Fifth International Workshop on Mathematical Aspects of Fluid and Plasma Dynamics (Maui, HI, 1998). Transport Theory Statist. Phys. 29 (2000), no. 3-5, 311-331.

[2] Coifman, R. and Meyer, Y., Commutateurs d'intégrales singulières et opérateurs multilinéaires. Ann. Inst. Fourier (Grenoble) 28 (1978), no. 3, xi, 177-202.

[3] Cordier, S. Grenier, E., Quasineutral limit of an Euler-Poisson system arising from plasma physics. Comm. Partial Differential Equations 25 (2000), no. 5-6, 1099-1113.

[4] Feldman, M. Ha, S-Y. and Slemrod, M., Self-similar isothermal irrotational motion for the Euler, Euler-Poisson systems and the formation of the plasma sheath. J. Hyperbolic Differ. Equ. 3 (2006), no. 2, 233-246.

[5] - A geometric level-set formulation of a plasma-sheath interface. Arch. Ration. Mech. Anal. 178 (2005), no. 1, 81-123.

[6] Germain, P., Masmoudi, N. and Shatah, J., Global solutions for 3D quadratic Schrödinger equations, Int. Math. Res. Not., 2009, no. 3, 414-432. 
[7] G Global solutions for the gravity water waves equation in dimension 3, preprint.

[8] Global solutions for 2D quadratic Schrödinger equations., preprint.

[9] Guo, Y., Smooth irrotational Flows in the large to the Euler-Poisson system in $R^{3+1}$ Commun. Math. Phys. 195, (1998), 249-265.

[10] Guo, Y. Tahvildar-Zadeh, A. S, Formation of singularities in relativistic fluid dynamics and in spherically symmetric plasma dynamics. Nonlinear partial differential equations (Evanston, IL, 1998), 151-161, Contemp. Math., 238, Amer. Math. Soc., Providence, RI, 1999.

[11] Guo, Z., Peng, L., and Wang, B., Decay estimates for a class of wave equations, J. Funct. Anal. 254 (2008), no. 6, 1642-1660.

[12] Gustafson, S., Nakanishi, K. and Tsai, T.P. Global dispersive solutions for the GrossPitaevskii equation in two and three dimensions. Ann. IHP 8 (2007), no. 7, 1303-1331.

[13] Scattering theory for the Gross-Pitaevskii equation in three dimensions. Commun. Contemp. Math. 11 (2009), no. 4, 657-707.

[14] John, F. Plane Waves and Spherical Means, Applied to Partial Differential Equations, reprint,

[15] Kato, T., The Cauchy problem for quasilinear symmetric systems, Arch. Ration. Mech. Anal. $58,(1975), 181-205$.

[16] Liu, H., Tadmor, E. Critical thresholds in 2D restricted Euler-Poisson equations. SIAM J. Appl. Math. 63 (2003), no. 6, 1889-1910 (electronic). 35Q35 (76X05)

[17] Liu, H. Tadmor, E., Spectral dynamics of the velocity gradient field in restricted flows. Comm. Math. Phys. 228 (2002), no. 3, 435-466.

[18] Muscalu, C., Paraproducts with flag singularities. I. A case study. Rev. Mat. Iberoam. 23 (2007), no. 2, 705-742.

[19] Muscalu, C., Pipher, J., Tao, T., Thiele, C., Multi-parameter paraproducts. Rev. Mat. Iberoam. 22 (2006), no. 3, 963-976.

[20] Peng, Y. Wang, S. Convergence of compressible Euler-Maxwell equations to compressible Euler-Poisson equations. Chin. Ann. Math. Ser. B 28 (2007), no. 5, 583-602.

[21] Peng, Y. Wang, Ya-Guang, Boundary layers and quasi-neutral limit in steady state EulerPoisson equations for potential flows. Nonlinearity 17 (2004), no. 3, 835-849.

[22] Shatah, J., Normal forms and quadratic nonlinear Klein-Gordon equations. Comm. Pure Appl. Math. 38 (1985), No 5, 685-696.

[23] Sideris, T. Formation of singularities in three-dimensional compressible fluids. Commun. Math. Phys. 101, (1985), 475-485.

[24] Stein, E. Harmonic analysis: real-variable methods, orthogonality, and oscillatory integrals, volume 43 of Princeton Mathematical Series. Princeton University Press, Princeton, NJ, 1993. With the assistance of Timothy S. Murphy, Monographs in Harmonic Analysis, III.

[25] Tao, T., Nonlinear dispersive equations, local and global analysis. CBMS. Regional Conference Series in Mathematics, 106. Published for the Conference Board of the Mathematical Science, Washington, DC; by the American Mathematical Society, Providence, RI, 2006. ISBN: 0-8218-4143-2.

[26] Texier, B., WKB asymptotics for the Euler-Maxwell equations. Asymptot. Anal. 42 (2005), no. 3-4, 211-250.

[27] Texier, B., Derivation of the Zakharov equations. Arch. Ration. Mech. Anal. 184 (2007), no. $1,121-183$.

[28] Wang, D. Global solution to the equations of viscous gas flows. Proc. Roy. Soc. Edinburgh Sect. A 131 (2001), no. 2, 437-449.

[29] Wang, D. Wang, Z. Large BV solutions to the compressible isothermal Euler-Poisson equations with spherical symmetry. Nonlinearity 19 (2006), no. 8, 1985-2004.

E-mail address: guoy@cfm.brown.edu Benoit.Pausader@math.brown.edu 